\title{
Development of inductive generalization with familiar categories
}

\author{
Anna V. Fisher • Karrie E. Godwin • Bryan J. Matlen
}

Published online: 4 March 2015

(C) Psychonomic Society, Inc. 2015

\begin{abstract}
Inductive generalization is ubiquitous in human cognition. In the developmental literature, two different theoretical accounts of this important process have been proposed: a naïve theory account and a similarity-based account. However, a number of recent findings cannot be explained within the existing theoretical accounts. We describe a revised version of the similarity-based account of inductive generalization with familiar categories. We tested the novel predictions of this account in two reported studies with 4-year-old children $(N=57)$. The reported studies include the first short-term longitudinal investigation of the development of children's induction with familiar categories, and it is the first study to explore the role of individual differences in semantic organization, general intelligence, working memory, and inhibition in children's induction.
\end{abstract}

Keywords Concepts and categories · Induction $\cdot$ Knowledge

Inductive generalization is the ability to make inferences from examples. This ability appears early in human development: at 3 months of age infants can generalize whether a novel object belongs to a previously seen category (Bomba \& Siqueland, 1983; Quinn, Eimas, \& Rosenkratz, 1993); at 10 months of age infants can make generalizations about observable object properties (Baldwin, Markman \& Melartin, 1993; Rakison \& Poulin-Dubois, 2002); by 24 months of age children can generalize known labels to novel objects (Booth \& Waxman, 2002; Jones \& Smith, 1998; Smith, Jones, \& Landau, 1996);

A. V. Fisher $(\triangle) \cdot$ K. E. Godwin

Department of Psychology, Carnegie Mellon University, 5000

Forbes Ave., 335-I Baker Hall, Pittsburgh, PA 15213, USA

e-mail: fisher49@andrew.cmu.edu

B. J. Matlen

WestEd, Oakland, CA 94612, USA and by preschool age children can make generalizations about unobservable object properties (Fisher, Matlen, \& Godwin, 2011; Gelman \& Markman, 1986; Sloutsky \& Fisher, 2004).

In this paper, we focus on the last kind of generalization: children's ability to make inferences about novel unobservable object properties. This kind of generalization is important to study, because it is ubiquitous in human cognition: for example, we can infer that a newly encountered banana is edible rather than having to learn this property anew with each encounter. Two alternative perspectives have been proposed for how children make such inferences: a naïve theory account (Gelman \& Markman, 1986; Markman, 1989) and a similarity-based account (Sloutsky \& Fisher, 2004; 2012a, b). We briefly describe these perspectives and demonstrate that they cannot account for all of the extant findings on children's inferences of unobservable object properties. We then propose a new theoretical framework that builds on the strengths of the extant accounts and present findings from two experiments that provide support for this framework.

\section{Theoretical accounts of inductive generalization in childhood}

Naïve theory account

In their seminal study, Gelman and Markman (1986) asked preschool-age children and college students to make inferences about natural kind items when perceptual information was ambiguous or conflicted with category membership (Sloutsky \& Fisher, 2004; Fisher, 2007). Labels were used to communicate category information: participants were asked whether a rock shared a nonobvious property with a stone or chalk. The overall selection rate of the category match items was above chance, both in preschool children and college students. These findings led the researchers to conclude that 
even young children make inferences based on naïve theories, in this case in the domain of biology. According to this account, young children hold a belief (or a naïve theory) that natural kind objects that belong to the same category will share a number of unobservable properties. Therefore, when making inferences, both children and adults first identify category membership of items under consideration and then generalize a known property to items of the same kind. Furthermore, this account suggests that children rely on category membership even when it conflicts with perceptual similarity. Finally, Gelman and Markman (1986) suggest that the ability to make category-based inferences is not a product of development and learning; instead, children are "initially biased" to recognize that labels denote categories and make inferences on the basis of shared category membership (p. 207), an idea that has become highly influential in the literature (Booth \& Waxman, 2002; Gelman \& Coley, 1990; Graham, Kilbreath, \& Welder, 2004; Jaswal \& Markman, 2007). Overall, this account emphasizes children's early competence in making category-based inferences and the striking similarities between inductive inferences made by very young children and adults.

\section{Perceptual similarity account}

In contrast to the two-step account of inductive inference suggested by the naïve theory approach, Sloutsky and Fisher (2004) proposed a one-step similarity-based account called SINC (for Similarity, Induction, Naming, and Categorization). According to SINC, children make inferences on the basis of the overall similarity of presented entities computed over all perceived object features, including visual and auditory attributes. A mathematical model that accompanied this theoretical account successfully captured the pattern of findings reported by Gelman and Markman (1986). Specifically, when visual features of the stimuli were ambiguous (e.g., the target matched one of the test objects on the shape dimension and the other test object on the texture and color dimensions; for details see Fisher, 2007), shared auditory features (e.g., identical linguistic labels) increased perceived similarity between the target item and category match. In this case, an inference could be label-based without necessarily being categorybased. Several related findings suggest that children rely primarily on perceptual features of objects (but not category membership information) to make inferences well beyond the preschool years, possibly until 7 to 9 years of age (Badger \& Shapiro, 2012; Fisher \& Sloutsky, 2005; Sloutsky, Kloos, \& Fisher, 2007).

Both accounts described above have considerable empirical support. However, when one considers all extant evidence, it is clear that each account encounters distinct challenges. The naïve theory account emphasizes early competence in making category-based inferences and continuity in the patterns of inductive generalization from early childhood to adulthood. Yet, a number of recent studies have reported a marked agerelated decrease in reliance on perceptual similarity and a marked age-related increase in the use of object kind information in the course of inductive generalization (Badger \& Shapiro, 2012; Fisher, Matlen, \& Godwin, 2011; Godwin, Matlen, \& Fisher, 2013; Sloutsky, Kloos, \& Fisher, 2007). Such age-related differences in performance clearly pose a challenge to the naïve theory account.

Recall that the SINC account (Sloutsky \& Fisher, 2004; 2012a) predicts that children make inductive inferences based on the overall perceptual similarity of presented items. Challenges to this account stem from findings suggesting that even young children are not limited to making inferences solely on the basis of perceptual similarity (Chi, Hutchinson, \& Robin, 1989; Fisher et al., 2011; Inagaki, 1990; Godwin et al., 2013; Opfer \& Bulloch, 2007). For example, Inagaki (1990) observed that 5-year-old children's inductive inferences differed as a function of their experience: compared to children who never had a pet, children who had experience caring for a goldfish were more likely to make biologically plausible inferences about other animals (e.g., a frog).

More recently, in a series of studies we asked children to make inductive inferences when no useful perceptual information was provided: objects were described as "hiding" behind identical doors or trees, which prevented children from relying on the overlap in visual features, and category information was communicated by semantically similar labels (rather than by identical labels) that provided no featural overlap in auditory information (Fisher et al., 2011; Godwin et al., 2013). Under these task conditions, most 4 -year-old children struggled to make inferences and performed at chance. For example, when told about an unobservable property of a sheep, most preschoolers were equally likely to make an inference to either a lamb (a category match) or a cow (a lure). Note that all categories used in these studies were highly familiar to 4-year-old children, based on their near-ceiling performance in a task that required children to choose the labeled item among several lures. Chance performance of 4-year-old children on this induction task is at odds with the naïve theory account but fits well within the SINC account: in the absence of perceptual overlap (either in visual or auditory features) most preschoolers were not able to make systematic inferences. However, under the same task conditions most 5- to 6-year-olds and nearly all 7-year-olds made categoryconsistent inferences (Fisher et al., 2011; Godwin et al., 2013). Findings suggesting that young children make inductive inferences on the basis of nonperceptual commonalities are difficult to reconcile with SINC, because it predicts that inferences are made strictly on the basis of perceptual similarity. Below, we describe a modified version of the similaritybased account that can explain this apparent contradiction as well as make novel predictions. 
Perceptual and representational similarity (PaRS) account of inductive generalization

We propose a new account called PaRS (for Perceptual and Representational similarity). The basic premise of PaRS is that one can distinguish two forms of featural similarity: perceptual and representational similarity. Perceptual similarity refers to the featural overlap in the input; it is computed online and in-the-moment for entities that are perceived directly. Note that an attentional weighting parameter can be used to specify why some features make a larger contribution to the overall similarity (i.e., based on differential saliency of various features) (Sloutsky \& Fisher 2004; 2012a). Nonetheless, perceptual similarity refers to the features of a directly observed entity.

Representational similarity, as the name implies, refers to the featural overlap in mental representations. Representational similarity includes properties that have been perceived directly in the past as well as properties that have come from conversations, books, and other indirect sources. For example, by going to a zoo one may observe directly that dolphins are large animals that live in water, and by reading a book about dolphins one may learn that dolphins give birth to live offspring and nurse their young with milk. All of the features that are encoded and stored in memory contribute to the representational similarity.

Within this theoretical approach, the notion of representational similarity is largely synonymous with the notion of semantic knowledge or semantic memory. It refers to the knowledge of objects acquired through experience (direct or indirect), including the knowledge of what things are called, how things look, where they may be found, and how they may be used. Importantly, our notion of representational similarity does not include knowledge that children may have "independent of experience" (Gelman \& Markman, 1986, p. 207). For example, knowledge of what a dog is includes semantic features (Clark, 1973), such as four legs, furry, and barks; some researchers have argued that it also may include an essentialist belief that "there is some unobservable property...- the essence" that makes something a dog" (Gelman, 2003, p. 7). The former but not the latter kind of knowledge is consistent with the idea of representational similarity put forth in this paper.

It should be noted that debates about the mechanisms of inductive generalization often are framed in terms of perception versus knowledge (Booth \& Waxman, 2002; Gelman \& Davidson, 2013; Gelman \& Markman, 1986; Sloutsky \& Fisher, 2004; Sloutsky, Kloos, \& Fisher, 2007). However, the juxtaposition of perception and knowledge is a false dichotomy (for similar arguments see Smith, Jones, Yoshida, \& Colunga, 2003). Knowledge can come in many forms: systems of associations are one form of knowledge (Smith et al., 2003); representations formed on the basis of perceptual experience are another form of knowledge (French, Mareschal, Mermillod, \& Quinn, 2004); essentialist beliefs are yet another form of knowledge (Gelman, 2003). In this light, making a distinction between different kinds of knowledge is important for advancing our understanding of cognitive development, including the development of inductive generalization. We suggest that representational similarity is a form of category knowledge that captures featural overlap among concepts in semantic memory (Sloman, 1993; Rogers \& McClelland, 2004).

According to PaRS, inductive generalization early in development is similarity-based, with both perceptual and representational similarity contributing to the overall similarity of presented entities; the probability of an inference is a function of the overall featural overlap between entities. Early studies of inductive generalization concluded that "by $2 \frac{1}{2}$ years, children expect categories to promote rich inductive inferences... and they can overlook conflicting perceptual appearances in doing so" (Gelman \& Coley, 1990, p. 802; see also Gelman \& Markman, 1986; Gelman \& Wellman, 1991). However, there is now overwhelming evidence that young children do not (and perhaps, cannot) ignore perceptual similarity when making inductive inferences (Badger \& Shapiro, 2012; Sloutsky, Lo, \& Fisher, 2001; Sloutsky \& Fisher, 2004; Sloutsky, Kloos, $\&$ Fisher, 2007). Moreover, the conclusion that perceptual similarity makes a large contribution to children's inferences has now been confirmed even by those researchers who initially opposed this idea (Graham, Booth, \& Waxman, 2012; Noles \& Gelman, 2012; c.f. 2012b). One of the goals of the present paper is to document the influence of representational similarity on inductive generalization in preschool children. Invoking the notion of representational similarity can help to explain several inconsistent findings in the literature. These inconsistences are discussed below.

Adult models of similarity-based induction vs. SINC: Do they make the same predictions and Can they account for the same data?

The SINC model of inductive generalization in children (Sloutsky \& Fisher, 2004, 2012) and Sloman's (1993) feature-based model of inductive inference have been discussed as belonging to the same class of models, namely similarity-based models (Sloutsky \& Fisher, 2004). Sloman's connectionist model was explicitly designed to account for phenomena associated with category-based reasoning in adult participants (Osherson, Smith, Wilkie, Lopez, \& Shafir, 1990) based on the proportion of shared features. In adult studies of induction, participants are typically asked to judge the strength of two arguments; for instance, the argument "Flamingos have a right aortic arch, therefore blackbirds have a right aortic arch" is judged to be a stronger argument than "Bats have a left aortic arch, therefore blackbirds have a left aortic arch" 
(Osherson et al., 1990, p. 195). One way to arrive at the conclusion that the former argument is stronger than the latter argument is to compare the "category coverage" (Osherson et al., 1990) of these two arguments: in the former case both items (i.e., flamingo and blackbird) belong to the category "bird," which is more specific than the category "animal" encompassing both items in the latter argument (i.e., bat and blackbird). Alternatively, one can account for this finding by noting that flamingos and blackbirds have more features in common (e.g., lay eggs, have feathers and beaks, etc.) than bats and blackbirds (e.g., can fly) (Sloman, 1993).

As we discussed above, the majority of preschoolers are at chance in making inductive inferences with familiar categories in the absence of directly available perceptual information, whereas older children and adults are nearly at ceiling in making inferences to category match items (Fisher et al., 2011; Godwin et al., 2013). If SINC and Sloman's featurebased induction model represent the same class of models (i.e., similarity-based models), it is not clear why they can selectively account for the performance of only young children (SINC) or only older children and adults (the featurebased model). SINC and Sloman's feature-based model of induction are similar in that both explain inferences in terms of feature overlap versus appealing to "a stable hierarchical category structure that is describable without reference to the attributes or features" of categories (Sloman, 1993, p. 232). At the same time, these two models are clearly distinct in that the former takes as input perceptual similarity (i.e., overlap in directly observable features), whereas the latter is based on representational similarity (i.e., overlap in features stored in memory, including features that may have never been observed directly). We suggest that both perceptual and representational similarity play an important role in inductive generalization and that developmental changes in representational similarity can explain developmental changes in inductive generalization observed in prior studies.

Computational models of semantic development suggest that representational similarity undergoes dramatic changes between the preschool and the early school years (Hills, Maouene, Maouene, Sheya, \& Smith, 2009; Kemp \& Tenenbaum 2008; Lowe, 1997; Rogers \& McClelland, 2004). Specifically, concepts are organized early in development on the basis of a small number of salient features that support the formation of relatively undifferentiated groups. Similarity in habitat seems to be one such particularly salient feature, leading to the formation of "thematic" clusters (e.g., dolphin, whale, penguin, and alligator; Kemp \& Tenenbaum 2008; Rogers \& McClelland, 2004). However, development and learning concepts become increasingly differentiated into groups that capture a larger number of more subtle relationships, leading to the formation of "taxonomic" clusters. There is recent evidence that this developmental trajectory characterizes changes in representational similarity not only in computational models but also in human participants (Fisher, Godwin, Matlen, \& Unger, 2014; Unger, Fisher, \& MacLellan, 2014). Therefore, developmental changes in representational similarity explain why Sloman's feature-based model would provide a good fit to performance of older children (whose representational similarity of familiar categories is in many ways similar to that in adults) but not that of preschoolers (whose representational similarity of familiar categories is dramatically different from that of adults; Fisher et al., 2014; Unger at al., 2014).

It is important to note that category-based and similaritybased inferences often yield the same generalization. As stated above, category-based induction involves first identifying the category membership of observed entities and then making an inference on the basis of this categorization. In contrast, similarity-based inferences involve identifying items with the highest featural overlap (either in perceived or in representational similarity). Therefore, it is possible for an inference to be category-consistent in its outcome without being categorybased in its mechanism. We think that it is important to introduce this distinction between category-based and categoryconsistent inferences to avoid confusion between the outcomes and the mechanisms of inductive generalization. Thus, we use the term category-based inference to refer to the putative mechanism of inductive generalization and the term category-consistent inference to describe a possible outcome of inductive generalization.

\section{Inconsistencies in developmental trajectories}

As discussed, some studies suggest that children are unlikely to make category-consistent inferences until approximately 9 years of age (Badger \& Shapiro, 2012; Sloutsky, Kloos, \& Fisher, 2007), whereas other studies report that children can make such inferences by 6 years of age (Fisher et al., 2011; Godwin et al., 2013; Long et al. 2011). Aside from procedural differences among these studies, a potentially important distinction is that the former studies used novel artificial categories whereas the latter studies used real familiar categories.

When familiar categories are used in experiments, it is possible that representational similarity helps children make category-consistent generalizations that are not categorybased in the mechanism by which they were generated (i.e., the inference may not involve identifying the category membership of presented items). In contrast, when reasoning about novel artificial categories, children's inferences cannot be category-consistent in their outcome without being categorybased in their mechanism. Newly learned artificial categories are based on a small number of shared features and their representations are likely not sufficiently differentiated to support inferences based on representational similarity (Hills, et al., 2009; Kemp \& Tenenbaum 2008; Rogers \& McClelland, 2004). Therefore, inconsistencies in the developmental 
trajectory of inductive generalization that have emerged across different studies can be explained by the notion of representational similarity.

\section{Developmental changes in induction with familiar categories}

Recall that most 4-year-old children are unlikely to make category-consistent inferences about familiar objects in the absence of supporting perceptual similarity; however, the vast majority of 6-year-old children are able to make such inferences (Fisher et al., 2011; Godwin et al., 2013). Semantic organization that includes a single densely interconnected cluster (Hills et al., 2009) or several poorly differentiated clusters (Kemp \& Tenenbaum, 2008; Rogers \& McClelland, 2004) is arguably ill suited for supporting categoryconsistent inferences. For instance, if one has to predict whether a crocodile is more likely to share an internal property with an alligator or a penguin, this task is trivial if representational similarity between the concepts of crocodile and alligator is higher than between the concepts of crocodile and penguin. However, the same task becomes more challenging if representational similarity of all three concepts is approximately equivalent, which may be the case early in development (Kemp \& Tenenbaum, 2008). Therefore, it is likely that developmental changes in representational similarity of familiar categories can explain the emergence of categoryconsistent generalizations in the course of development.

\section{Summary}

The PaRS theoretical framework shares some important similarities with the existing accounts of inductive generalization in young children, but it also is distinct from these accounts. PaRS is similar to SINC (Sloutsky \& Fisher, 2004; 2012a, b) in that it is a similarity-based account that characterizes inductive inferences as a one-step process of identifying entities with the highest featural overlap. Unlike SINC and similar to the naivve theory account, PaRS does not assume that children's inferences are driven exclusively by perceptual similarity. However, unlike the naïve theory account, PaRS (1) posits no influences on inductive generalization that are "independent of experience" (Gelman \& Markman, 1986, p. 207), and (2) aims to understand the nature of developmental differences and developmental change in inductive generalization as opposed to emphasizing continuity in patterns of inductive generalization from childhood to adulthood.

\section{Novel predictions of the PaRS account}

In addition to being able to account for several inconsistencies in the literature, the PaRS account can generate new predictions. Recall that early in development, semantic representations are poorly differentiated but become increasingly differentiated in accordance with taxonomic relations in the course of learning and development (Fisher, et al., 2014; Hills, et al., 2009; Kemp \& Tenenbaum 2008; Rogers \& McClelland, 2004; Unger et al., 2014). For example, Unger et al. (2014) observed that preschoolers and kindergarteners were likely to group together items, such as whale, seaweed, and water lily (likely due to similarity in habitat); however, by second grade children grouped items largely in accordance with the plants-animals distinction.

The first novel prediction generated by the PaRS account is that poorly differentiated representations should be unlikely to support category-consistent inferences when lures are high in representational similarity to the target items. However, if lures have little to no overlap in representational similarity to the target items, it should be possible to elicit categoryconsistent inferences even in young children whose representations are still overall poorly differentiated. In other words, whether a child makes a category-consistent inference should be a function of the similarity ratio of each test item to the target, whether this similarity ratio describes perceptual similarity (Sloutsky \& Fisher 2004; 2012a, b) or representational similarity. Specifically, the PaRS account predicts that when representational similarity between the target item and category match is held constant (e.g., lamb-sheep), the probability of a category-consistent inference should be low when representational similarity between the target and lure is relatively high (e.g., lamb-cow); in contrast, the probability of a categoryconsistent inference should be high when representational similarity between the target and lure is low (e.g., lamb-clock). This prediction was tested in Experiment 1.

The second novel prediction of the PaRS account is that individual differences in representational similarity can help to explain individual variability in preschoolers' inductive inferences with familiar categories that has been documented in prior studies. Specifically, only a minority of children in this age group can be classified as category-consistent responders (Fisher et al., 2011; Gelman \& Markman, 1986; Godwin, et al., 2013). For example, in the Gelman and Markman (1986) study only $6 \%$ of children made category-consistent inferences when semantically similar labels were used to communicate category information and $31 \%$ of participants made category-consistent inferences when identical labels were used to communicate category information (Gelman and Markman, 1986, Table 6, p. 201). Recall that these studies used categories and labels that were highly familiar to preschoolers. Therefore, it is unclear why the majority of children did not provide category-consistent responses, particularly if children are initially biased towards category-based generalization.

As stated above, representational similarity changes as children learn an increasing number of attributes associated with different categories; and these changes in representational similarity result in semantic knowledge becoming 
increasingly organized in accordance with taxonomic relations (Fisher et al., 2014; Kemp \& Tenenbaum 2008; Rogers \& McClelland, 2004; Unger et al., 2014). Changes to representational similarity are driven by experience (both direct and indirect); therefore, semantic organization in individual children will vary based on both the available input and a child's ability to encode and store this input. In other words, even though taxonomic organization of semantic knowledge may be a common outcome of semantic development, individual children's development will proceed towards this outcome at a different pace. Therefore, we predict that some of the individual variability in the emergence of category-consistent inferences with familiar categories is due to individual differences in organization of semantic knowledge (i.e., representational similarity). We test this prediction in Experiment 2.

In addition to developmental differences in the organization of semantic knowledge, we expect that children's ability to deploy this knowledge in the course of inductive generalization is affected by individual differences in the ability to actively maintain representations in working memory and in inhibitory control. This prediction (which we test in Experiment 2) rests on the observation that the vast majority of induction tasks impose significant demands on at least one of these processes. Specifically, induction tasks often seek to ensure that children's performance is not an outcome of a simple matching strategy. As a result, most experimental paradigms introduce conflict between perceptual similarity and category membership (Gelman \& Markman, 1986; Long et al. 2011; Sloutsky \& Fisher, 2004) and thereby impose the need for inhibitory control to resolve the conflict between different sources of information. Other experimental paradigms remove perceptual similarity as a possible source of information (Fisher et al., 2011; Godwin et al., 2013; Fisher et al. in press), resulting in a task that imposes significant demands on working memory.

Age-related improvements in working memory and inhibitory control have been implicated in developmental accounts of category learning (Sloutsky, 2010) and analogical reasoning (Krawczyk, Morrison, Viskontas, Holyoak, Chow, Mendez, Miller, \& Knowlton, 2008; Morrison, Doumas, \& Richland, 2011; Richland, Morrison \& Holyoak, 2006); however, the relationship between individual differences in these processes and the emergence of category-consistent inferences has remained unexplored. In what follows, we discuss what role inhibitory control and representation maintenance-processes that are subserved by the areas within the pre-frontal cortex (Diamond, 2002; Miller \& Cohen, 2001) - may play in children's performance on inductive generalization tasks.

Relative immaturity of inhibitory control may contribute to children's performance on tasks that select items in such a way that perceptual similarity is in conflict with category membership (for examples of such tasks see Badger \& Shapiro, 2012;
Fisher, 2011; Gelman \& Markman, 1986; Gentner, 1978; Sloutsky \& Fisher, 2004; Sloutsky, Kloos, \& Fisher, 2007). Participants in these studies typically include children between 3 and 5 years of age, a period during which inhibitory control is known to undergo significant development (Akshoomoff, 2002; Carlson, 2005; Gerstadt, Hong, \& Diamond, 1994). Richland et al. (2006) suggested that ignoring perceptually compelling lures in favor of perceptually dissimilar category matches in higher-order reasoning tasks may be particularly challenging for young children in this age range. There is a great deal of evidence to support this assertion (Fisher 2011; Graham et al., 2012; Noles \& Gelman, 2012; Sloutsky \& Fisher, 2004; Sloutsky, et al., 2007).

Although it is clear that 3- to 5-year-old children have difficulty resolving conflict between different sources of information, it is not clear to what degree this is a limiting factor in children's inductive generalization. For example, Badger and Shapiro (2012) tested children ranging from 3 to 9 years of age in a triad induction paradigm with novel natural-kindlike bug categories. Children were introduced to two categories of bug-like creatures, both in their mature form as well as in their juvenile (i.e., larva) form. Children were shown that two distinct kinds of larvae grew up to become two distinct kinds of bugs; however, in the mature form, the two categories of bugs were more perceptually similar to each other than to their respective juvenile forms. On every trial, children watched a short movie depicting the transformation of the target item from its juvenile form into its mature form and were then asked to make a property projection from the target item to one of the two test items: a category match or a perceptual lure. There was a marked increase in the propensity towards category-based induction with age: among 3- to 4year-old children, only $13 \%$ were classified as category-based responders, with $65 \%$ of participants classified as similaritybased responders; whereas among 8- to 9-year-old children, $73 \%$ of participants were classified as category-based responders and only $20 \%$ as similarity-based responders. However, Badger and Shapiro (2012) found that in every age group children made fewer category-based inferences when high similarity distracters were present compared with lower similarity distracters. The researchers concluded that development of category-based induction is unlikely to stem from improvements in inhibitory control, as older children (with presumably more developed inhibitory control) showed a similar mild decrement in performance to younger children (with presumably much less developed inhibitory control).

Studies in which no useful visual information is presented seem to point to a similar conclusion: even when items are described as "hiding" behind identical objects and there is no perceptually salient lure that needs to be ignored, few preschoolers succeed in providing category-consistent inferences (Fisher et al., 2011; Godwin et al., 2013). Nonetheless, it is possible that maturation of the prefrontal cortex (PFC) and 
inhibitory control contribute to the development of categorybased inference in a way distinct from resolving conflict between perceptual similarity and category membership, particularly in tasks that rely on verbal descriptions of familiar objects. Specifically, it is possible that a relatively mature PFC is required to inhibit non-task-relevant representations and select task-relevant representations among multiple competing activated representations (for review see Kan \& ThompsonSchill, 2004). For example, when children are asked whether a sheep or a cow is more likely to have a property known to be true of a lamb, children may have difficulty in selecting between the category match (e.g., sheep) and the lure (e.g., cow) both of which have been activated by the task instructions, even if representations of these categories are sufficiently differentiated in the semantic similarity space to support category-consistent inferences.

It also has been suggested that rather than help to select among competing activated representations, the role of the PFC in controlled semantic access is to bias retrieval of taskrelevant semantic information by maintaining task representations in working memory (for a review see Badre \& Wagner, 2002). In other words, children may fail to make categoryconsistent inferences (even if representations of these categories are sufficiently differentiated to support such inferences) due to the limited ability to hold task-relevant information and manipulate this information in working memory (e.g., to evaluate whether the target item is more similar to the category match or the lure). Therefore, another factor that may be able to account for the variability in emergence of categoryconsistent inferences in young children is individual differences in working memory.

\section{Present research}

As we discussed above, research on the development of children's ability to make category-consistent inferences with familiar categories points to a protracted developmental course as well as to a high degree of individual variability early in development. However, the sources of this individual variability as well as sources of developmental change remain unclear. The goals of the studies described in this manuscript were twofold. The first goal was to examine the putative role of representational similarity in inductive generalizations of preschool-age children. Towards this goal, we tested the prediction of the PaRS framework that children's ability to make categoryconsistent inferences with familiar categories is a function of not only the representational similarity between the target item and category match, but also between the target item and the lure (Experiment 1).

The second goal of the present work was to elucidate possible sources of individual variability in the emergence of category-consistent inferences with familiar categories, with the focus on the putative role of semantic development, working memory, and inhibitory control. To investigate these questions, we used a short-term longitudinal design (Experiment 2): we assessed children's inductive inferences at the beginning and at the end of their second year in preschool, the period during which many children begin to make categoryconsistent inferences with familiar categories (Fisher et al., 2011; Gelman \& Markman, 1986; Godwin et al., 2013). To obtain measures of inhibitory control and working memory, we selected several tasks commonly used in the literature; we also obtained a measure of children's general intelligence using the Wechsler Preschool and Primary Scale of Intelligence (Wechsler, 1989).

To obtain measures of individual differences in semantic organization, we used a recently developed Semantic Space task (Fisher, et al., in press). In the Semantic Space task, participants are asked to represent the semantic similarity of animal dyads in two-dimensional space. The distance between animal pairs is taken as a measure of representational similarity. Physical distance has been successfully used in prior research as an indicator of psychological proximity (Goldstone, 1994; Howard \& Howard, 1977). Unlike the Multidimensional Scaling studies in which children are free to arrange the items along any desired dimension of similarity, we explicitly asked children to put animals of similar kind close together on the board. On different trials of the task, participants were asked to arrange the same target animal (e.g., sheep) on the game board in relation to a semantically-similar item (e.g., lamb) and an unrelated item (e.g., swan). Taking the difference between the placement of semantically related and unrelated items allows us to derive an individual index of semantic differentiation. This index can then be used to examine the relation between children's semantic differentiation and propensity to make category-consistent inferences.

\section{Experiment 1}

Experiment 1 tested the prediction of the PaRS account that category-consistent inferences with familiar categories in preschool children are a function of not only the representational similarity between the target item and category match but also between the target item and the lure. Towards this goal, for each target and category match pair (e.g., rat-mouse) we calibrated two different lures, a semantically close lure (e.g., skunk) and a semantically distant lure (e.g., spoon). We then asked 4-year-old children to select the test item (i.e., the category match or the lure) that shares a property with the target item. We predicted that the rate of category-consistent inferences should be higher with semantically distant lures than with semantically close lures. 
Participants

Participants were recruited from a laboratory school of a private university in a Northeastern city. Participants were predominantly from middle to high SES households. One child was tested but excluded from the analyses reported below due to limited comprehension of the English language. The final sample in Experiment 1 consisted of 15 4-year-old children (7 males, 8 females; Mage $=4.41$ years, $S D=0.27$ ).

\section{Design and procedure}

Participants completed an Inductive Generalization task, in which they were asked to generalize a property of a target item to one of the two test items, a category match or a lure. Items were not depicted but instead were described as hiding behind identical doors. Prior research shows that this procedure can be successfully used with preschool-age children to test inductive generalization (Fisher et al., 2011; Godwin et al., 2013). See Fig. 1 for a schematic depiction of the task. To-be-generalized properties were two-syllable blank predicates (e.g., omat).

Each target item (e.g., rat) was paired with a category choice (e.g., mouse) and a lure. Half of the trials used semantically close lures (i.e., lures were from the same ontological class as the target and category choice; e.g., skunk) and the other half of the trials used semantically distant lures (i.e., lures were from a different ontological class; e.g., spoon). All target items, category matches, and close lures in Experiment 1 were animals, and all distant lures consisted of artifacts. See Table 1 for a full list of linguistic stimuli used in Experiment 1.

A separate group of adult participants $(N=10$, MAge $=$ 19.56) provided semantic similarity ratings for the items used in Experiment 1. These similarity ratings confirmed that the target items were viewed as more semantically similar to the category matches $(M=5.96)$ than to either type of lure ( $M$ Close Lures $=4.38, M$ Distant Lures $=1.08$ ), both paired-samples $t \mathrm{~s}>3.46, p \mathrm{~s}<0.05$. At the same time, Close lures were judged as more semantically similar to the target items than Distant lures, paired-samples $t(4)=11.91, p<0.0001$.

Experiment 1 used a within-subjects design, such that each participant was presented with the same target item and category choice item twice: once in the context of a Close lure and once in the context of a Distant lure. For example, in the Close Lure condition the experimenter asked whether a mouse or a skunk had the same property as a rat; and in the Distant Lure condition the experimenter asked whether a mouse or a spoon had the same property as a rat. The lures were matched in syllable length to the category choice items. Both types of trials (i.e., Close and Distant Lures) were intermixed and presented in one of two pseudo-random orders within a single testing session; the pseudo-random orders were created with the constraints that (1) trials containing the same target item could not occur in a row, and (2) no more than two trials of the same type could occur in a row. The order in which the experimenter named the test items within a triad (i.e., the category match first or the lure first) was counterbalanced across trials. There were 5 trials in each lure type condition for a total of 10 trials.

Finally, at the conclusion of the induction task, a vocabulary check was administered to all participants to ensure that children were familiar with the labels used in the study. The vocabulary check was modeled after the Peabody Picture Vocabulary Test (Dunn \& Dunn, 1997). Children were presented with four pictures on each trial and were asked to point to the object named by the experimenter.

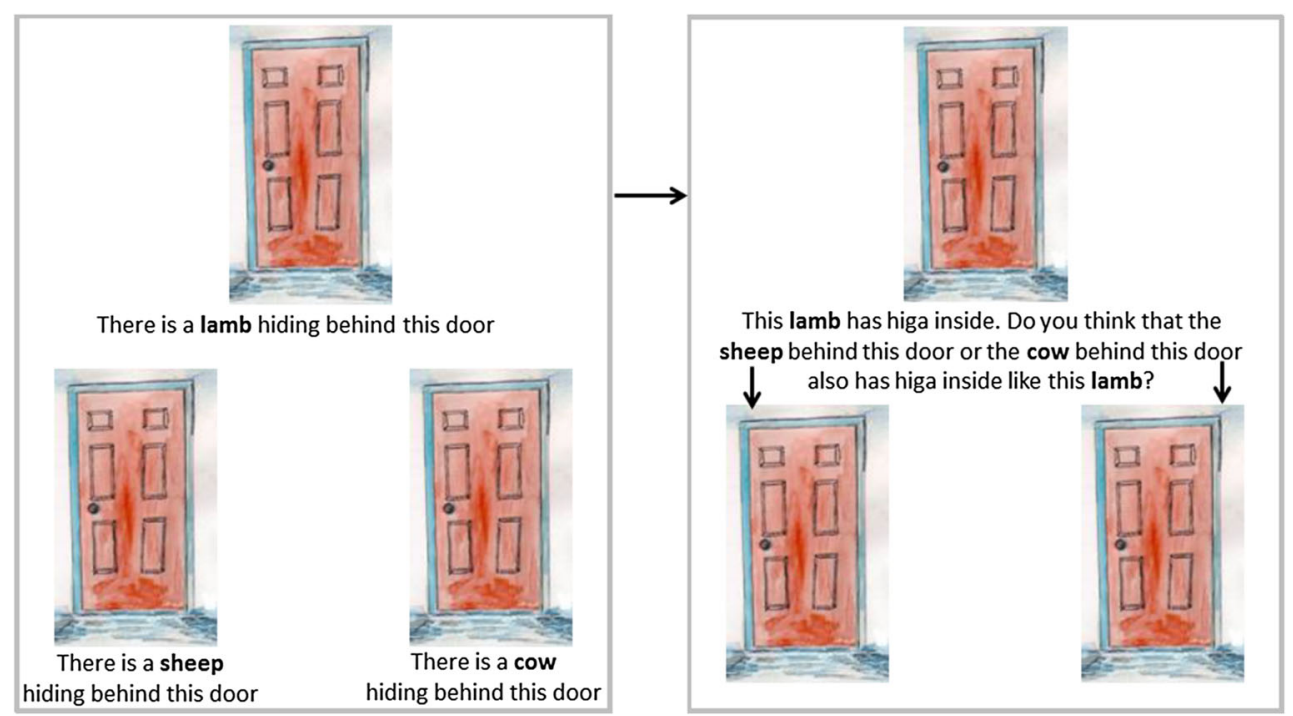

Fig. 1 Schematic depiction of the Inductive Generalization task in the Close Lure condition. Note that all instructions were given verbally by the experimenter 
Table 1 Complete list of linguistic stimuli used in Experiment 1

\begin{tabular}{lllll}
\hline Target & Category match & Close lure & Distant lure & Property \\
\hline Lamb & Sheep & Cow & Clock & Higa \\
Rat & Mouse & Skunk & Spoon & Omat \\
Crocodile & Alligator & Hippopotamus & Refrigerator & Erwin \\
Dolphin & Whale & Seal & Sock & Lignin \\
Caterpillar & Butterfly & Ladybug & Telephone & Troxel \\
\hline
\end{tabular}

Results and discussion

The results of the vocabulary check indicated that children's accuracy approached ceiling $(M=0.98)$, suggesting that children were highly familiar with the linguistic stimuli used in the Inductive Generalization task. There were no significant differences in the vocabulary accuracy scores on the different types of items $(M$ Targets $=0.97, M$ Category Match $=1.0, M$ Close Lures $=0.97, M$ Distant Lures $=1.0$ ), all paired-samples $t \mathrm{~s}<1, n s$.

On the Inductive Generalization task, the average proportion of category match choices was higher in the Distant Lure condition $(M=0.75, S D=0.29)$ than in the Close Lure condition $(M=0.58, S D=0.30)$, paired-samples $t(14)=2.61, p<$ 0.05 , Cohen's $d=0.58$. Moreover, the average proportion of category match choices was above chance in the Distant Lure condition, one-sample $t(14)=3.21, p<0.005$ but not different from chance in the Close Lure condition, one-sample $t(14)=$ $1.06, p>0.30$.

To investigate individual patterns of responses, we classified participants as category-consistent responders if they chose the category match for at least four of five trials (or $80 \%$ ) in each lure type condition. ${ }^{1}$ In the Close Lure condition, six children were classified as category-consistent responders and nine children as mixed responders; in the Distant Lure condition, ten children were classified as categoryconsistent responders and five children were classified as mixed responders. Of the six children who were classified as category-consistent responders in the Close Lure condition, only one child was classified as a mixed responder in the Distant Lure condition; in contrast, of the nine children classified as mixed responders in the Close Lure condition, five were classified as category-consistent responders in the Distant Lure condition. A McNemar change test for correlated proportions suggested that these differences were significant, $p<0.05$.

\footnotetext{
${ }^{1}$ The criterion of at least four category-consistent responses on five trials is not statistically different from chance responding, binomial $p=.18$. If a more stringent criterion is used to classify a child as a category-consistent responder (i.e., five category-consistent responses on five trials, binomial $p=.03)$, the reported outcomes of the McNemar change test remain significant, $p<.01$.
}

The outcomes observed in the Close Lure condition of Experiment 1 replicate the previous findings suggesting that most preschoolers fail to make systematic category-consistent inferences when lure items come from the same ontological category as the target items (Fisher et al., 2011; Gelman \& Markman, 1986; Godwin et al., 2013; Matlen et al., under review). The results in the Distant Lure condition provide novel evidence supporting the predictions of the PaRS account. Recall that the target and category choice items were identical across both lure type conditions. Therefore, these findings support the prediction that the probability of a category-consistent response is a function of the overall semantic similarity of all presented items. At the same time, the pattern of results in Experiment 1 is at odds with the extant developmental accounts of inductive generalization. The naive theory can explain children's above-chance performance in the Distant Lure condition, but it cannot explain chance performance in the Close Lure condition. The naïve theory account of inductive generalization specifically predicts that young children should make category-consistent inferences with triads that include close lures (e.g., rock-stone-chalk, bunny-rabbit-squirrel in Gelman \& Markman, 1986); therefore, the results of the Close Lure condition are clearly at odds with the predictions of the naïve theory approach. SINC has the opposite challenge, because it is able to account for children's chance performance in the Close Lure condition (i.e., on this account, children are not expected to make systematic inferences in the absence of useful perceptual information) but not for the systematic category-consistent inferences in the Distant Lure condition. In contrast, PaRS can account for the full pattern of results observed in Experiment 1.

\section{Experiment 2}

The findings from Experiment 1 support the prediction of the PaRS account that the probability of a category-consistent inference in preschool-age children is a function of representational similarity of presented items. Experiment 2 will further test this prediction using an individual difference approach. Specifically, Experiment 2 tests the prediction that individual differences in children's inductive generalization with familiar categories are a function of individual differences in organization of semantic knowledge. Experiment 2 also examined the prediction that children's ability to deploy semantic knowledge in the course of inductive generalization is influenced by individual differences in working memory and inhibitory control.

\section{Participants}

In Experiment 2, 43 participants were 4-year-old children from a laboratory preschool at a private university in a 
Northeastern city. Participants were from predominantly middle to high SES households; none of the participants in Experiment 2 participated in Experiment 1. All children were in their second year of preschool. One child was excluded from the analyses, because this child moved during the school year and was not available for testing in the middle and end of the school year. The final sample consisted of 42 children: 20 females and 22 males. At Time 1, the children were on average aged 4.33 years $(S D=0.28$, range $3.94-4.98$ years); at Time 2 , the children were on average aged 4.77 years $(S D=$ 0.31, range 4.29-5.36 years). Note that a subset of data from Time 1 was reported separately (Fisher et al., in press).

\section{Design and procedure}

Each child participated in a total of 12 testing sessions during the course of their second year in preschool between the months of September and May. Multiple tasks were often administered within the same testing session, and some tasks were administered at multiple time points (see Table 2 for a detailed description of the task administration schedule). Children were tested individually in a quiet room adjacent to their classroom by a trained research assistant; each testing session lasted no longer than 15 minutes. The task battery used in this study is described below.

\section{Inductive generalization task}

The visual stimuli and procedure in the Inductive Generalization task were identical to those in Experiment 1. We used three target items from Experiment 1 (i.e., alligator, rat, and

Table 2 Schedule of task administration in Experiment 2

\begin{tabular}{|c|c|c|}
\hline Session & Tasks & Time period \\
\hline 1 & IQ: Block design \& information subtests & \multirow{6}{*}{$\begin{array}{l}\text { September - } \\
\text { November }\end{array}$} \\
\hline 2 & $\begin{array}{l}\text { Inductive generalization Time } 1 \mathrm{~A} \\
\text { Picture identification Time } 1\end{array}$ & \\
\hline 3 & $\begin{array}{l}\text { IQ: matrix reasoning, vocabulary, } \\
\text { \& coding subtests }\end{array}$ & \\
\hline 4 & $\begin{array}{l}\text { Inductive generalization Time 1B } \\
\text { Forward word span task }\end{array}$ & \\
\hline 5 & $\begin{array}{l}\text { IQ: picture concepts, word reasoning, symbol } \\
\text { search subtests }\end{array}$ & \\
\hline 6 & $\begin{array}{l}\text { Semantic space Time } 1 \\
\text { Backward word span task }\end{array}$ & \\
\hline 7 & Track-it & \multirow{3}{*}{$\begin{array}{l}\text { January - } \\
\quad \text { March }\end{array}$} \\
\hline 8 & Day-night task & \\
\hline 9 & Flanker task & \\
\hline 10 & $\begin{array}{l}\text { Inductive generalization Time } 2 \mathrm{~A} \\
\text { Picture identification Time } 2\end{array}$ & \multirow[t]{3}{*}{ March-May } \\
\hline 11 & Semantic space Time 2 & \\
\hline 12 & Inductive generalization Time 2B & \\
\hline
\end{tabular}

lamb) and added three target items referring to inanimate natural kinds and three target items referring to artifacts (see Table 3 for the full list of linguistic stimuli used in the induction task in Experiment 2). Similar to Experiment 1, all triads in Experiment 2 contained a target item, a category match, and a lure. Trials in the induction task were administered in one of two counter-balanced orders.

To maximize individual differences in performance in Experiment 2 , we selected lures to be moderately semantically similar to the target items (i.e., less similar than the close lures but more similar than the distant lures in Experiment 1). The same adult participants who provided semantic similarity ratings for Experiment 1 also provided semantic similarity ratings for Experiment 2. The category match items were rated as more semantically similar to the target items $(M=6.30)$ than lures $(M=2.83)$, paired-samples $t(8)=10.76, p<0.0001$. At the same time, the medium similarity lures chosen for Experiment $2(M=2.83)$ were rated as more semantically similar to targets than the distant lures $(M=1.08)$ but less similar than the close lures $(M=4.38)$ in Experiment 1, both pairedsamples $t \mathrm{~s}>8.02, p \mathrm{~s}<0.0001$.

The Inductive Generalization task was administered twice at Time 1 (i.e., Time 1A and 1B) and twice at Time 2 (i.e., Time 2A and 2B): 1) to obtain a more stable estimate of children's performance and 2) to ensure that improvement in category-based reasoning from Time 1 to Time 2 cannot be attributed solely to children's increased familiarity with the task. The average delay between the repeated administrations of the induction task was 4.43 days $(S D=3.34)$ at Time 1 and 20.69 days $(S D=3.32)$ at Time 2 . The average delay between the administration of this task at Time 1 and Time 2 was 5.25 months $(S D=0.90)$.

At Times 1A and 2A, immediately following the inductive generalization task a vocabulary check was administered to ensure that children were familiar with the labels used in the

Table 3 Linguistic stimuli used in the inductive generalization task in Experiment 2

\begin{tabular}{|c|c|c|c|}
\hline Target & Category-choice & Lure & Property (blank predicate) \\
\hline \multicolumn{4}{|l|}{ Artifacts } \\
\hline Shoe & Boot & Car & Troxel \\
\hline Rug & Carpet & Window & Koski \\
\hline Sofa & Couch & Cup & Creighan \\
\hline \multicolumn{4}{|c|}{ Inanimate natural kinds } \\
\hline Rock & Stone & Grass & Higa \\
\hline Hill & Mountain & Flower & Erwin \\
\hline Sea & Ocean & Apple & Manchin \\
\hline \multicolumn{4}{|l|}{ Animals } \\
\hline Alligator & Crocodile & Butterfly & Omat \\
\hline Rat & Mouse & Fish & Lignin \\
\hline Lamb & Sheep & Frog & Matlen \\
\hline
\end{tabular}


study. The vocabulary check was modeled after the Peabody Picture Vocabulary Test (Dunn \& Dunn, 1997). Children were presented with four pictures on each trial and were asked to point to the object named by the experimenter. The results indicated that children's accuracy on the vocabulary check approached ceiling $(M$ Time $1=.92$ and $M$ Time2 $=0.96$ ), suggesting that children were highly familiar with the linguistic stimuli used in the induction task.

\section{Semantic space task}

The semantic space task served as a measure of children's semantic organization. Following Howard and Howard (1977), we used unpainted 1" wooden cubes as stimuli to encourage children to use their knowledge about kinds rather than rely on perceptual similarity. Children were asked to arrange the animals (represented by wooden cubes) on a twodimensional game board consisting of a 9x9 grid (Fig. 2).

Linguistic stimuli included 24 pairs of animal names. The critical stimuli consisted of 18 pairs of animal names that could be classified into one of three categories: 1) category match (i.e., items that were closely related taxonomically, such as crocodile-alligator), 2) habitat match (i.e., items that share a common habitat, such as crocodile-fish), and 3) unrelated dyads (e.g., crocodile-grasshopper). During the game, each target item was paired with three different test items: the category match, the habitat match, and the unrelated item. Additionally, we used 6 pairs of animal names as filler items (e.g., zebra-turkey). The filler items were structurally identical to the unrelated dyads, but they did not use the same target animals as the critical stimuli. The filler trials were included solely to encourage children to utilize the entire game board

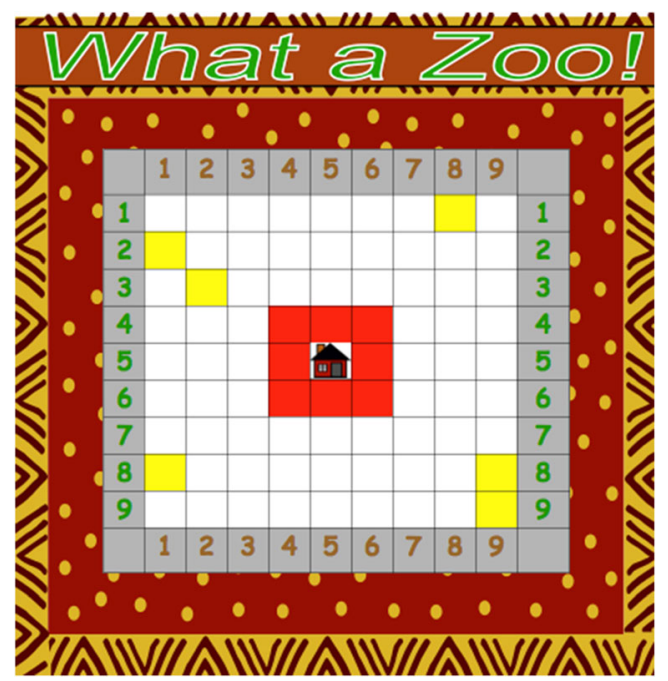

Fig. 2 Schematic depiction of the Semantic Space game board. Squares highlighted in red indicate the location of the critical trials; squares highlighted in yellow mark the location of the filler trials. Note that in the experiment the location of the critical and filler trials were not marked and all squares on the board were white (see the description of the procedure below). Therefore, children's performance on the filler items was not scored and was not used in the data analyses reported below. A full list of linguistic stimuli in the Semantic Space task is provided in Table 4.

Similar to Howard and Howard (1977), we asked children to help a fictional character (in our case, Zibbo the zookeeper) to arrange his animals in the zoo by placing similar animals close together. However, unlike the previous studies in which similarity was unconstrained, we asked children to consider taxonomic relatedness: children were asked to place animals similar in kind close together. At the beginning of the task, the experimenter introduced the game and provided the participants with two examples. The experimenter placed two game pieces representing a bunny and a rabbit on adjacent squares on the game board and explained that these animals should be placed close together because "they are the same kind of thing"; the experimenter then placed two game pieces representing a dog and a shark eight squares apart and explained that these animals should be placed far apart, because they are "not the same kind of thing." On each test trial, the experimenter showed the participant where the zookeeper put the target animal (e.g., "The zookeeper put the mouse here"), handed the participant the second game piece, and asked him or her to identify where the test item should be placed (e.g., "Where do you think the hippo should go?"). The participant's response was recorded to calculate the distance between the placement of the target and test item. The game board was cleared at the end of each trial.

Placement of the 18 critical trials (i.e., category match, habitat match, and unrelated dyads) was pseudo randomized to eight central squares (marked in red in Fig. 2). The central squares were utilized for the critical trials to approximately equate the range of possible distances between the test item and the target across trials. Each of the eight central squares was used at least twice and no more than three times. The six

Table 4 Linguistic stimuli for the semantic space task

\begin{tabular}{clll}
\hline Target & $\begin{array}{l}\text { Category- } \\
\text { match }\end{array}$ & Habitat-match & Unrelated \\
\hline $\begin{array}{c}\text { Critical trials } \\
\text { Crocodile }\end{array}$ & Alligator & Fish (water animals) & Grasshopper \\
Chick & Hen & Goat (farm animals) & Goldfish \\
Lamb & Sheep & Horse (farm animals) & Swan \\
Whale & Dolphin & Octopus (water animals) & Elephant \\
Monkey & Gorilla & Parrot (jungle/wild & Chipmunk \\
Mouse & Rat & Pig (farmals) animals) & Hippo
\end{tabular}

Filler pairs

$\begin{array}{lll}\text { Zebra/Turkey } & \text { Bear/Snake } & \text { Panther/Turtle } \\ \text { Tiger/Butterfly } & \text { Frog/Lion } & \text { Giraffe/Seal }\end{array}$


filler trials were randomly assigned to one of the remaining 24 squares to encourage participants to use the entire game board. The animal dyads were presented in one of two pseudo randomized orders, which were created with the following constraints: one filler trial was presented after every three critical trials, at least three trials were required in between target repeats, and at least two trials were presented in between category match dyads. The presentation order was counterbalanced across participants.

Participants' responses on the Semantic Space task were scored using a variant of the city block distance measure, which computes absolute distances between coordinates of a pair of objects. ${ }^{2}$ Specifically, raw scores were calculated for each trial by adding the number of squares occupied by the game pieces (i.e., two if the participant placed the blocks on different squares) and the number of squares between the target and test item (based on the shortest route between the two game pieces barring diagonal movement). A Semantic Space difference score was calculated by subtracting the average score for the category match dyads from the average score for the habitat match and unrelated dyads. Positive difference scores indicate that participants put category match items closer together than habitat match and unrelated items. The maximum difference score a participant could obtain on this task was 9 or 10 (depending on the exact placement of the target block); therefore, the maximum average score in this task was 9.5. Difference scores approaching zero indicate that participants did not reliably discriminate among category match, habitat match, and unrelated dyads. Most of the analyses reported below were conducted on the difference scores rather than the raw scores to eliminate the influence of individual placement biases. For example, some participants may have a tendency to place all items relatively close together, whereas other participants may have a tendency to place all items relatively far apart. Of primary interest in this study was whether children would place the game blocks closer together for the category match dyads than for habitat match and unrelated dyads, rather than the absolute placement of the blocks. The average delay between the administration of this task at Time 1 and Time 2 was 5.58 months $(S D=0.92)$.

\section{General intelligence test}

Children completed 8 subtests of the Wechsler Preschool and Primary Scale of Intelligence (WPPSI; Wechsler, 1989): Block Design, Matrix Reasoning, Picture Concepts, Information, Vocabulary, Word Reasoning, Symbol Search, and Coding. Children's performance on these subtests provided a measure of general intelligence (Full-scale IQ). The subtests also can be combined to obtain estimates of children's verbal

\footnotetext{
${ }^{2}$ All of the reported findings remain unchanged if Euclidian distance is used instead of the city block distance.
}

reasoning and comprehension (Verbal IQ); an estimate of fluid reasoning, spatial processing, and visual-motor integration (Performance IQ); and an estimate of children's ability to quickly and accurately scan, sequence, and discriminate simple visual information (Processing Speed Quotient or PSQ). The intelligence test was administered in 3 separate testing sessions due to the schools' requirement that children could not be absent from the classroom for longer than 20 minutes and could only be tested once a day. For a given child, all of the subtests in the WPPSI battery were administered within 5 school days barring individual student absences. The order of the task administration was as follows: 1) IQ testing session 1 (Block Design and Information); 2) IQ testing session 2 (Matrix Reasoning, Vocabulary, and Coding); 3) IQ testing session 3 (Picture Concepts, Word Reasoning, and Symbol Search).

\section{Working memory tasks}

It is common to distinguish between two functions of Working Memory: short-term storage and manipulation of information (Tulsky, Carlozzi, Chevalier, Espy, Beaumont, \& Mungas, 2013). In this study, we included two measures that probed short-term storage (i.e., Forward Word Span and Track-It Memory) and one measure that probed short-term storage and manipulation of information (i.e., Backward Word Span).

In the Forward and Backward Word Span tasks stimuli consisted of 128 common, one-syllable count nouns selected from the MacArthur Communicative Development Inventory (Dale \& Fenson, 1996). From this list of count nouns, two word lists of equal length were created: Words were arranged in each list such that they were not phonologically or thematically related to an adjacent word. These two lists then comprised the stimuli for the Forward and Backward Word Span tasks. Children were given either the Forward Word Span task in List 1, and then the Backward Word Span task in List 2, or vice versa. In the Forward Word Span task, children were asked to recite the words in the same order in which they were presented; in the Backward Word Span task children were asked to repeat the words in the opposite order.

The word span tasks were administered in two separate testing sessions. All children completed the Forward Word Span task first. The average delay between testing sessions was 3.81 days. Children were first given two practice trials in which they repeated two words in either forward or backward order (depending on the task being tested). The experimenter provided feedback if the child did not correctly complete the practice trials. After the practice trials, children began the test trials. The test trials were divided into 5 sets. The initial set size included lists of two words. The maximum set size was 6 words. Each set included three trials of the same list length. Set size increased monotonically. In order to move on to the next set size, children needed to correctly recite the words in the specified order on two of the three trials. Incorrect 
responses included omitting a word or reciting the list in the wrong order. Children's score was determined by the longest set size the child was able to recite correctly.

We used the memory component of the Track-It Task as a measure of nonverbal working memory (Fisher, Thiessen, Godwin, Kloos, \& Dickerson 2013). In this task, children were asked to watch a target object moving amidst six distracters on a $3 \times 3$ grid on a computer screen. The target and distracter objects moved along a random trajectory for approximately 10 seconds before disappearing. Children were asked to report the last grid location visited by the Target object. On every trial, after reporting the last location visited by the Target, children completed a memory task. In the memory task, children were presented with nine objects and were asked to identify the object that served as the Target on the just-completed trial. There were ten experimental trials in the Track-It task.

\section{Inhibitory control tasks}

Two common measures of response inhibition were included in the assessment battery: the Day-Night task (Gerstadt, et al., 1994) and a child-friendly adaptation of the Flanker task (Rueda, Fan, McCandliss, Halparin, Gruber, Lercari, \& Posner, 2004). In the Day-Night Task, children were shown a set of cards depicting the sun and the moon. Children were asked to provide a verbal response that conflicts with the presented image (e.g., if the child was shown a picture of the sun, the correct response would be "night." Conversely if the child was shown a picture of the moon, the correct response would be "day"). The dependent measure in this task was proportion of correct responses. The task consisted of 16 trials (the moon and the sun were presented 8 times each). Two presentation orders were created: The trials were randomized for order 1 and the sequence was reversed for order 2 .

For the child-friendly adaptation of the Flanker Task, children were presented with arrays of fish on a computer screen. Children were asked to feed the center fish by pressing either the left or right button; the correct response depended upon the direction the center fish was facing. The center fish was surrounded by four other fish (two on each side). The surrounding fish could be congruent (e.g., swimming in the same direction as the center fish) or incongruent (e.g., swimming in the opposite direction as the center fish). Neutral trials also were presented in which the central fish appears in isolation (i.e., not flanked by other fish). There was a total of 48 trials: 16 neutral trials, 16 incongruent trials, and 16 congruent trials. For the purpose of the analyses reported below, we used the Flanker Accuracy Difference score (calculated by subtracting each child's accuracy for the Incongruent trials from the Neutral trials).

It is well-known that many inhibitory control tasks place substantial demands on working memory, making it difficult to obtain a "pure" measure of inhibitory control (Best \& Miller, 2010; Simpson \& Riggs 2005). Keeping this issue in mind, we selected inhibitory control tasks that are likely to provide a measure of inhibitory control relatively uncontaminated by working memory demands. In the case of the Flanker task, the memory demands are minimal as children need to only remember the instruction to press the arrow pointing in the direction of the central fish. The relationship between working memory and inhibitory control is more complex in the case of the Day/Night task; however, prior research suggests children's performance on this task cannot be explained solely by appealing to working memory. Specifically, when Simpson and Riggs (2005) eliminated the presence of a predominant response (believed to probe inhibitory control) from the Day/Night task, developmental improvement typically observed on this task between 3 and 5 years of age nearly disappeared, suggesting that a large proportion of variability in performance on the Day/Night task can be attributed to inhibitory control.

\section{Results and discussion}

This section is divided into three parts. In Part I, we present analyses of children's performance on individual tasks. In Part II, we examine the relationship between semantic differentiation and emergence of category-consistent responding in the induction task. In Part III, we used automatic model discovery and structural equation modeling techniques to identify relationships among all predictor variables measured in this study and performance on the induction task.

Part I: analysis of performance on individual tasks

A summary of performance on all tasks administered in this study is provided in Table 5.

\section{Inductive generalization task}

The average proportions of category-consistent inferences at Time $1 \mathrm{~A}$ and $1 \mathrm{~B}$ were nearly identical $(0.62$ and 0.63 , respectively) and significantly correlated, $r=0.50, p<0.005$. This finding is important, because it suggests that any improvement in children's category-based induction observed from Time 1 to Time 2 cannot be attributed to children's increased familiarity with the task but rather stems from children's increased tendency to make spontaneous category-consistent inferences.

The average proportion of category-consistent scores at Time 2B (0.81) was higher than at Time 2A (0.71), pairedsamples $t(41)=3.31, p<0.005$. Responses on the categorybased reasoning task were strongly correlated at Time $2 \mathrm{~A}$ and $2 \mathrm{~B}, r=0.69, p<0.0001$, suggesting that children's patterns of responses were highly stable at Time 2 . 
Table 5 Summary of performance on the task battery in Experiment 2 for the three types of responders on the Induction task

\begin{tabular}{|c|c|c|c|}
\hline & $\begin{array}{l}\text { Category- } \\
\text { consistent at } \\
\text { Time } 1(n=10)\end{array}$ & $\begin{array}{l}\text { Category- } \\
\text { consistent at } \\
\text { Time } 2(n=18)\end{array}$ & $\begin{array}{l}\text { Not-category- } \\
\text { consistent }(n=14)\end{array}$ \\
\hline Age (at Time 1) & $4.32(0.28)$ & $4.35(0.32)$ & $4.29(0.24)$ \\
\hline $\begin{array}{l}\text { Induction at } \\
\text { Time } 1\end{array}$ & $0.93(0.07)$ & $0.56(0.13)$ & $0.49(0.13)$ \\
\hline $\begin{array}{l}\text { Induction at } \\
\text { Time } 2\end{array}$ & $0.87(.13)$ & $0.90(0.07)$ & $0.49(0.14)$ \\
\hline $\begin{array}{l}\text { Semantic space } \\
\text { difference } \\
\text { scores Time } 1\end{array}$ & $2.68(1.81)$ & $1.21(2.03)$ & $0.72(1.30)$ \\
\hline $\begin{array}{l}\text { Semantic space } \\
\text { difference } \\
\text { scores Time } 2\end{array}$ & $3.22(2.41)$ & $2.76(1.94)$ & $1.27(1.76)$ \\
\hline Full-scale IQ & $119.9(10.63)$ & $108.0(13.6)$ & $99.7(13.41)$ \\
\hline Verbal IQ & $122.2(20.89)$ & $107.05(32.11)$ & $96.5(24.56)$ \\
\hline Processing speed & $105.5(7.09)$ & $102.5(14.54)$ & $93.64(13.80)$ \\
\hline Performance IQ & $115.5(8.37)$ & $107.55(14.45)$ & $103.93(17.99)$ \\
\hline FWS & $3.50(1.4)$ & $3.44(1.04)$ & $2.28(1.32)$ \\
\hline BWS & $1.50(1.08)$ & $1.89(0.96)$ & $0.43(0.85)$ \\
\hline Track-It memory & $0.73(0.23)$ & $0.58(0.32)$ & $0.16(0.15)$ \\
\hline Day/Night & $0.70(0.34)$ & $0.79(0.23)$ & $0.46(0.27)$ \\
\hline $\begin{array}{l}\text { Flanker Accuracy } \\
\text { Difference } \\
\text { scores }\end{array}$ & $0.29(0.16)$ & $0.40(0.22)$ & $0.31(0.36)$ \\
\hline
\end{tabular}

For the remaining analyses, induction scores were averaged across Time $1 \mathrm{~A}$ and $1 \mathrm{~B}$ and across Time $2 \mathrm{~A}$ and $2 \mathrm{~B}$ to yield average category-consistent inference scores for Time 1 and Time 2. The rate of category-consistent responding at Time $1(M=0.627)$ was above chance, $t(41)=19.25, p<$ 0.001 . To examine the possible effect of trial type, induction scores from Time 1 were submitted to repeated measures Analysis of Variance (ANOVA) with trial type (Animals, Inanimate Natural Kinds, or Artifacts) as the within-subject factor. Effect of trial type was not significant at Time 1, $F(2,82)=$ $1.89, p>0.15$, suggesting that children exhibited comparable performance on all three kinds of trials (MAnimals $=0.66$, MInanimate Nat.Kinds $=0.64$, MArtifacts $=0.58$ ). Indeed, the proportion of category-consistent responses was above chance on all three trial types, all one-sample $t \mathrm{~s}>2.03, p \mathrm{~s}<0.05$.

The proportion of category-consistent responses at Time 2 $(M=0.76)$ also was above chance, $t(41)=22.23, p<0.001$, and higher than at Time 1, paired-samples $t(41)=3.63, p<$ 0.005 , Cohen's $d=0.60$. Recall that the latter finding cannot be attributed simply to children having experience with performing the same task, because scores were nearly identical at Time 1A and Time 1B. Therefore, this finding points to a developmental increase in the propensity towards categoryconsistent reasoning. To examine possible effects of trial type, induction scores from Time 2 were submitted to repeated measures ANOVA with trial type (Animals, Inanimate Natural Kinds, or Artifacts) as the within-subject factor. At Time 2 the effect of trial type was significant, $F(2,82)=6.53, p<0.005$, $\eta_{\mathrm{p}}{ }^{2}=0.23$. Pairwise comparisons indicated that the rate of category-consistent responses was higher on Animal and Inanimate Natural Kind trials $(M=0.78$ and $M=0.81$, respectively) than on the Artifact trials $(M=0.69), p<0.05$. At the same time, the average proportion of category-consistent responses was above chance on all three types of trials, all onesample $t \mathrm{~s}>4.1, p \mathrm{~s}<0.0001$. Importantly, the proportion of category-consistent responses increased on all three types of trials from Time 1 to Time 2, all paired-samples $t \mathrm{~s}>2.06, p \mathrm{~s}<0.05$.

To investigate individual patterns of responses, participants were classified as either category-consistent or mixed responders. A category-consistent responder was defined as a participant who selected the category match on at least $75 \%$ of the trials across the two testing sessions within each time point (i.e., across Time 1A and 1B; across Time $2 \mathrm{~A}$ and $2 \mathrm{~B}$; binomial $p<0.02$ ). In line with prior findings in the literature, this analysis revealed that only a minority of participants consistently provided category-consistent responses at Time 1: 10 of 42 children ( $24 \%$ of the sample). The majority of children who were classified as category-consistent responders at Time 1 also were classified as category-consistent responders at Time 2 (9/10 children), suggesting that our classification was based on genuine differences in children's performance. At the same time, among the 32 children who were classified as mixed responders at Time 1, 18 children were classified as category-consistent responders at Time 2 . Therefore, at Time 2 the majority of children were classified as categoryconsistent responders: 27 of 42 children (64\% of the sample; Fig. 3). The number of children who shifted from being mixed responders to being category-consistent responders $(n=18)$ was significantly greater than the number of children who shifted in the opposite direction $(n=1)$, McNemar's change test $\chi^{2}(1)=15.21, p<0.001$, providing further support to the developmental trend observed in the analysis of overall means. Finally, a sizable minority of children were classified as mixed responders at both Time 1 and Time 2: 14 of 42 children ( $33 \%$ of the sample). This finding is consistent with prior cross-sectional studies suggesting that the ability to make category-consistent inferences with familiar categories develops gradually between 4 and 6 years of age and that there is a sizable minority of children who are not yet categoryconsistent responders by the time of entry into kindergarten (Fisher, et al., 2011; Godwin et al., 2013).

Note that children's performance at Time 1 in Experiment 2 , which used lures of medium semantic similarity to the targets, yielded $62.7 \%$ of category-consistent responses. Similarly-aged children in Experiment 1 (i.e., 4.41 years of age in Experiment 1 compared to 4.33 years of age at Time 1a in Experiment 2), made category-consistent inferences with close lures $58 \%$ of the time and with distant lures $75 \%$ of 

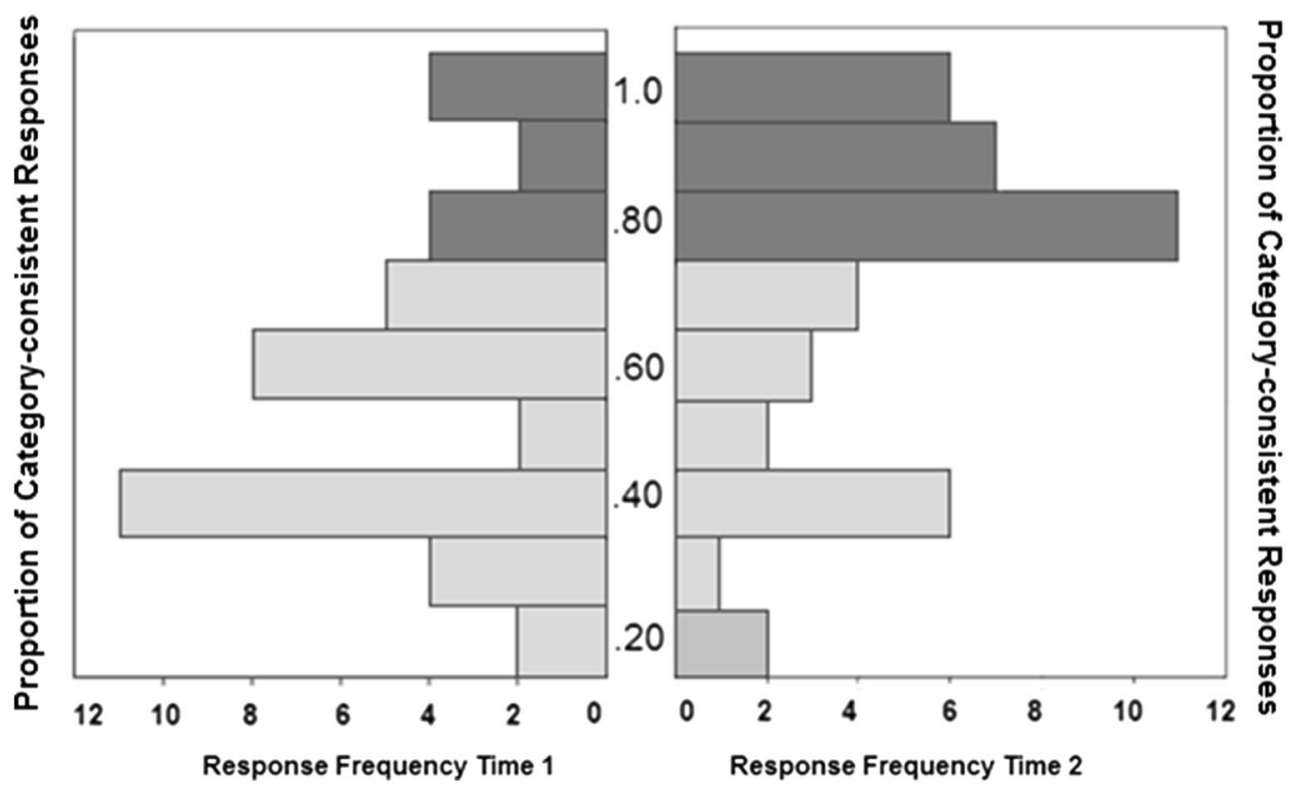

Fig. 3 Distributions of responses in the Inductive Generalization task at Time 1 and Time 2

the time. Several factors complicate direct comparisons between Experiments 1 and 2; however, it is noteworthy that children's performance with medium similarity lures was numerically lower than performance with distant lures, but higher than performance with close lures, providing further support to the hypothesis tested in Experiment 1.

\section{Semantic space task}

At Time 1, children on average placed the category match dyads 4.34 squares apart, habitat match dyads 5.83 squares apart, and unrelated dyads 5.64 squares apart. These scores were submitted to repeated measures ANOVA with trial type as the within-subjects factor. This analysis revealed a main effect of trial type, $F(2,82)=16.09, p<0.0001, \eta_{\mathrm{p}}{ }^{2}=$ 0.282 . Planned comparisons indicated that this effect stemmed from performance on the category match dyads being different from performance on both unrelated and habitat match dyads, $p<0.05$; no other comparisons were significant. Because there was no significant difference between children's placements of habitat match dyads and unrelated dyads $(p>0.41)$, children's responses on the habitat match dyads and unrelated dyads were collapsed into a single category of noncategory match dyads $(M=5.74)$. We than calculated the Semantic Space Difference scores by subtracting for each participant the mean placement of category match dyads from the mean placement of the noncategory-match dyads. ${ }^{3}$ At Time 1, the mean Semantic Space Difference score averaged across all

\footnotetext{
${ }^{3}$ All of the reported findings remain unchanged if we calculate the difference scores based on placements of unrelated dyads and category match dyads at both Time 1 and Time 2 .
}

participants was 1.40 , significantly different from zero, onesample $t(41)=4.82, p<0.0001$. Difference scores above zero indicate that children placed category match dyads closer together than noncategory-match dyads suggesting some degree of semantic differentiation. At the group level, semantic differentiation was relatively weak; however, some children showed high semantic differentiation at Time 1, with Semantic Space Difference scores ranging from -2.58 to 5.67.

At Time 2, children on average placed the category match dyads 3.49 squares apart, habitat match dyads 5.72 squares apart, and unrelated dyads 6.01 squares apart. There was no change in the placement of the habitat match dyads and unrelated dyads from Time 1 to Time 2, both paired-samples $t \mathbf{s}<1$, $n s$. However, children placed category match dyads closer together at Time $2(M=3.49)$ than at Time $1(M=4.34)$, paired-samples $t(41)=2.89, p<0.005$, Cohen's $d=0.59$.

Semantic Space scores at Time 2 were submitted to repeated measures ANOVA with trial type as the within-subjects factor. This analysis revealed a main effect of trial type, $F(2$, $82)=38.81, p<0.0001, \eta_{\mathrm{p}}{ }^{2}=0.473$. As at Time 1 , this effect was driven by the differences in placement of category match trials and other types of trials, both $p \mathrm{~s}<0.05$. Because there was no difference in the placement of habitat matches and unrelated dyads, $p>0.25$, similar to Time 1 children's responses on these trials were collapsed into a single category of noncategory-match dyads $(M=5.87)$ at Time 2 . We then calculated the Semantic Space Difference scores by subtracting for each participant the mean placement of category-match dyads from the mean placement of the noncategory-match dyads. At Time 2, the mean Semantic Space Difference score averaged across all participants was 2.37 (with scores ranging from -1.08 to 7.58 ), significantly 
different from zero, one-sample $t(41)=7.24, p<0.0001$. Furthermore, the Semantic Space Difference scores were significantly higher at Time 2 than at Time 1, paired-samples $t$ $(41)=2.56, p<0.05$, Cohen's $d=0.48$, suggesting an increase in semantic differentiation between the beginning and end of children's second preschool year.

\section{Wechsler preschool and primary scale of intelligence}

The Wechsler Preschool and Primary Scale of Intelligence (WPPSI) composite score and subscales have a mean of 100 $(S D=15)$; scores between 90 and 110 are considered average (Wechsler, 1989). In the present study, children's Full-scale IQ was in the average range: $M=108.07, S D=14.72$ (scores ranged from 67 to 140). Similarly, children's average subscale scores were within average range: Verbal IQ $M=107.14, S D$ $=24.55$ (scores ranged from 53 to 145), Performance IQ $M=$ 108.24, $S D=14.97$ (scores ranged from 70 to 135 ), and Processing Speed Quotient $M=100.26, S D=13.53$ (scores ranged from 73 to 128).

\section{Measures of executive functioning}

In the Forward Word Span (FWS) task, children on average correctly recalled 3.07 words $(S D=1.33$; scores ranged from 0 to 5). In the Backward Word Span (BWS) task, children on average correctly recalled 1.31 words ( $S D=1.14$; scores ranged from 0 to 3 ). Consistent with prior findings in the literature (Chen \& Stevenson, 1988), children's FWS scores were significantly higher than their BWS scores, paired-samples $t(41)=8.51$, $p<0.0001$, Cohen's $d=1.42$.

Average accuracy on the memory check questions in the Track-It task was $M=0.48(S D=0.34$; scores ranged from 0 to 1). There were nine response options on the memory check; therefore, chance performance is $M=0.11$. Children's average accuracy on this task was significantly above chance, onesample $t(41)=7.01, p<0.0001$.

On the Flanker task, accuracy data were missing from one participant due to experimenter error. For the remaining participants accuracy on the Neutral, Congruent, and Incongruent trials was as follows: $M$ Neutral $=0.69(S D=0.21$; scores ranged from 0 to 0.94$), M$ Congruent $=0.65(S D=0.25$; scores ranged from 0 to 1$)$, and MIncongruent $=0.35(S D=0.24$; scores ranged from 0 to 0.81 ). Accuracy was significantly higher on the Congruent and Neutral trials than on the Incongruent trials, both paired-samples $t \mathrm{~s}(40)>8.079, p \mathrm{~s}<0.0001$; there was no difference in accuracy between the Congruent and Neutral trials, paired-samples $t(40)=1.52, p>0.13$. We calculated Flanker task Accuracy Difference scores by subtracting each participant's accuracy on Incongruent trials (on which the central stimulus faces in the direction opposite to the flanking stimuli) from the accuracy on the Neutral trials (on which the central stimulus was not flanked). This analysis yielded an average Flanker task Accuracy Difference score of $0.34(S D=0.26$; scores ranged from -0.44 to 0.75$)$, above zero, one-sample $t(41)=8.26, p<0.0001$.

Data from one participant were missing for the Day/Night task due to the child's refusal to comply with the experimenter's instructions. The remaining participants averaged 0.66 of correct responses on the Day/Night task $(S D=0.30$; scores ranged from 0 to 1$)$, above chance ( 0.50$)$, one-sample $t$ $(40)=3.44, p<0.005$.

Part II: relationship between induction performance and semantic differentiation

Children's tendency to choose the category match item on the Inductive Generalization task was positively correlated with children's Difference scores on the Semantic Space task at both Time 1 and Time 2. Specifically, induction performance at Time 1 correlated with semantic differentiation at Time $1(r=0.461$, $p<0.005)$ and induction performance at Time 2 correlated with semantic differentiation at Time $2(r=0.368, p<0.05)$. In other words, greater semantic differentiation of familiar concepts corresponded to a greater tendency to choose category match items on the induction task.

The remainder of the analyses in this section focus on different response profiles in the inductive generalization task and semantic differentiation at Time 1 and Time 2. Note that several children reached the ceiling or near-ceiling level of performance on the inductive generalization task at Time 1; therefore, it would be inappropriate to analyze children's change scores from Time 1 to Time 2: for some children the change scores would be 0 , because they were already at ceiling at Time 1 , whereas for other children the change scores would be 0 , because the children performed at chance at both Time 1 and Time 2. Instead, we separated children into different groups based on their response profiles: children were classified as category-consistent responders if they chose the category match item on at least $75 \%$ of the trials across the two testing sessions within each time point; otherwise, children were classified as mixed responders. Based on this criterion, we separated children into the following three groups of responders: Category-consistent at Time 1 (CCT1), Category-consistent at Time 2 (CCT2), and Notcategory-consistent at Time 1-2 (NCCT1-2). Group CCT1 consisted of children who were classified as categoryconsistent responders at Time $1(n=10)$; as stated previously, most of these children (9/10) were classified as categoryconsistent responders at Time 2. Group CCT2 consisted of children who were classified as mixed responders at Time 1 but as category-consistent responders at Time $2(n=18)$. Finally, Group NCCT1-2 consisted of children who were classified as mixed responders at both Time 1 and Time $2(n=14)$.

The Semantic Space task was the only predictor task that was administered at the beginning and end of children's 
second preschool year. In this section, we report how developmental changes in children's performance on the task of inductive generalization were related to developmental changes in children's performance on the Semantic Space task. The number of children in Group CCT1 was fairly low $(n=10)$; therefore, there may be insufficient statistical power to detect meaningful differences. The analyses below use the alphalevel of 0.1 for all comparisons involving Group CCT1 and alpha-level of 0.05 for all other comparisons.

At Time 1, children in Group CCT1 had higher Semantic Space Difference scores than children in Group CCT2, independent-samples $t(26)=1.91, p=0.068$, Cohen's $d=$ 0.76 , and children in Group 3, independent-samples $t(22)=$ $3.10, p<0.001$, Cohen's $d=1.24$. At the same time, there were no significant differences in semantic differentiation between children in Groups CCT2 and NCCT1-2 at Time 1, independent-samples $t(30)<1, n s$. In contrast, at Time 2 the Semantic Space Difference scores of children in Group CCT2 were significantly higher than those of children in Group NCCT1-2, independent-samples $t(30)=2.21, p<$ 0.05 , Cohen's $d=0.79$, and comparable to the scores of children in Group CCT1, $t(26)<1, n s$.

From Time 1 to Time 2, children in Group CCT1 did not show a significant increase in their Semantic Space Difference scores, although recall that these children already showed evidence of moderate semantic differentiation at Time 1, pairedsamples $t(9)<1, n s$. Children in Group NCCT1-2 also had no significant change in their Semantic Space Difference scores from Time 1 to Time 2, paired-samples $t(13)=1.09, p>0.29$, suggesting that these children had fairly low semantic differentiation both in the beginning and end of their second preschool year. In contrast, children in Group CCT2 had significantly higher Semantic Space Difference scores at Time 2 than at Time 1, paired-samples $t(17)=2.53, p<0.05$, Cohen's $d=0.77$. In fact, the Semantic Space Difference scores of children in Group CCT2 at Time 2 were comparable to the Semantic Space Difference scores of children in Group CCT1 at both Time 1 and Time 2, independent-samples $t \mathrm{~s}<$ $1, n s$. These findings are summarized in Fig. 4.

Overall, the analyses above suggest that only children with moderately high Semantic Space Difference scores at the beginning of the preschool year and children whose Semantic Space Difference scores became moderately high by the end of the preschool year were able to provide spontaneous category-consistent responses on the inductive generalization task. At the same time, children whose Semantic Space Difference scores remained low throughout their second year in preschool were not yet able to make spontaneous categoryconsistent inferences at the end of the school year.

Part III: relationship among All predictor variables and Children's performance on the inductive generalization task

Analyses described in this section aim to identify possible causal relations in the measured set of variables through automatic model discovery and Structural Equation Modeling (SEM) (Spirtes, Glymour, \& Scheines, 2000). Prior to performing these analyses, we sought to reduce the number of observed variables ostensibly probing the same latent variables through exploratory factor analysis (Miyake et al., 2000); the outcomes of this analysis are described in the Appendix. Based on the outcomes of the exploratory factor analysis, we calculated a composite measure of Working Memory (by transforming the raw scores into z-scores and averaging the z-scores across the FWS, BWS, and Track-It tasks) and a composite measure of Inhibition (by transforming the raw scores into z-scores and averaging the z-scores across the Day/Night and the Flanker tasks). These composite scores were used in the analyses described below.

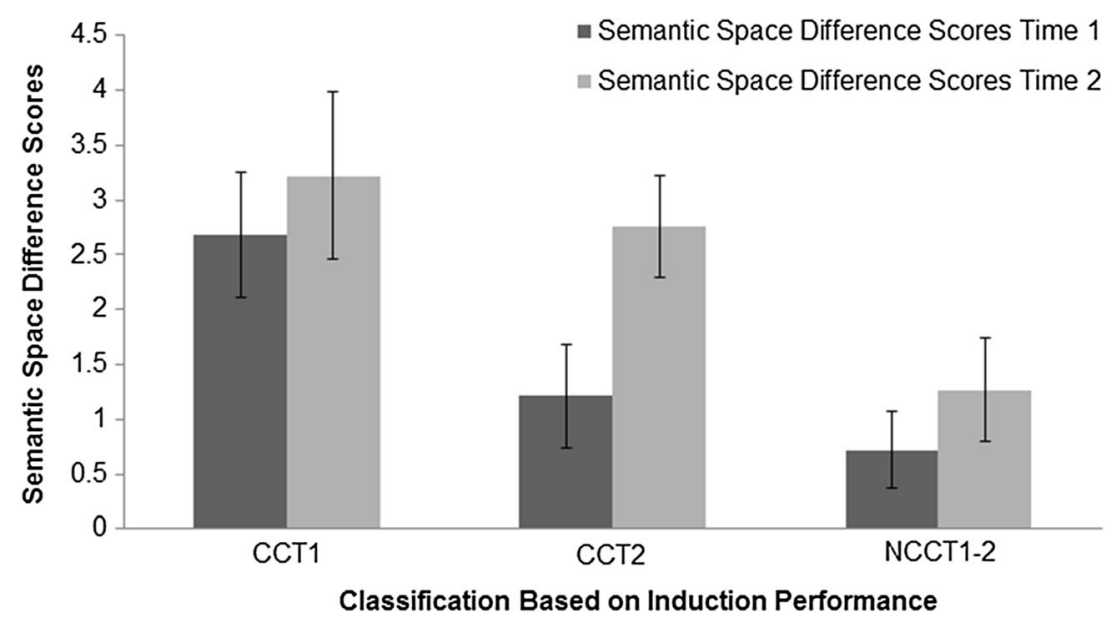

Fig. 4 Change in Semantic Space Difference scores from Time 1 to Time 2 in children classified as the following types of responders on the induction task: category-consistent at Time 1 (CCT1); category-

consistent at Time 2 (CCT2); non-category-consistent at Time 1 and Time 2 (NCCT1-2). Error bars represents the standard errors of the mean 


\section{Identifying possible causal relations at time 1}

The intercorrelations among all variables used in the analyses below are presented in Table 6 . The analyses described in this section were performed using causal inference algorithms implemented in TETRAD IV software. TETRAD can be used for automatic discovery of causal models from data, as well as evaluation and parameterization of causal models (Spirtes, et al., 2000; Scheines et al., 1998). Casual models are represented by directed acyclic graphs, which specify a joint probability distribution. TETRAD belongs to the class of constraint-based algorithms of causal inference (Spirtes, et al., 2000); as such, it tests constraints that true patterns of causal connectivity obey with regards to observed data. Specifically, TETRAD algorithms search over possible causal structures of the observed data based on the information about conditional independencies of the variables under consideration.

First, we performed automatic search for relations among the following set of variables: Induction scores at Time 1, Full-scale IQ, Semantic Space Differences scores at Time 1, composite Inhibition scores, and composite Working Memory scores. We did this using the Fast Causal Inference (FCI) search algorithm (Spirtes, 2001) implemented in TETRAD IV. The output of this search algorithm is a partial ancestral graph, which gives partial information about which variables are (or are not) direct or indirect causes and effects of other variables. An edge between two variables implies that the variables are not independent (conditional on any set of the measured variables). The following kinds of edges are possible in partial ancestral graphs: 1) an unmarked (by either an arrowhead or a circle) tail of an edge from $X$ to $Y$ suggests that $\mathrm{X}$ is a cause of $\mathrm{Y} ; 2$ ) an edge from $\mathrm{X}$ to $\mathrm{Y}$ with an arrowhead directed into $\mathrm{Y}$ suggests that $\mathrm{Y}$ is not a cause of $\mathrm{X} ; 3$ ) an edge with two arrowheads (pointing both to $\mathrm{X}$ and to $\mathrm{Y}$ ) suggest that there is an unrecorded common cause of $X$ and $Y$; 4) an edge marked with an open circle on the end suggests that the search algorithm cannot determine whether there should or should not be an arrowhead at that edge end. Whenever the automatic search algorithm determines that there is an edge between two variables but is unable to determine the direction of the causal relation, there is a class of possible causal models consistent with the automatically discovered structure. Possible causal models can then be parameterized and evaluated using SEM implemented in TETRAD IV software.

Prior to performing the analyses described in this section, all scores were standardized by transforming raw scores into z-scores. FCI search discovered a set of relations in the data at Time 1, but was unable to discover the directionality of these relations (Fig. 5). The following variables were found to be nonindependent from each other: Working Memory and Fullscale IQ; Full-scale IQ and Semantic Space scores at Time 1; and Semantic Space scores at Time 1 and Induction scores at Time 1.

The structure of the data automatically discovered with the FCI algorithm is consistent with several possible causal models. Using TETRAD IV software we parameterized and evaluated three causal models consistent with the automatically discovered structure; these models are presented in Fig. 6 (Models 1-3). In constructing these models, we made the assumption that Working Memory contributes to Full-scale IQ: it is plausible to assume that a simpler process (Working Memory) contributes to performance on a complex set of tasks (Full-scale IQ; hence the causal arrow from Working Memory to Full-scale IQ for Models 1-3 in Fig. 6). The statistical summary evaluating these models includes a goodness of fit chisquare test. It is important to note the null hypothesis for the goodness of fit chi-square test is that the model is not different from data. Therefore, large chi-square values corresponding to $p$ values $<0.05$ suggest that the model is not consistent with the data and should be rejected. Small chi-square values corresponding to $p$ values $>0.05$ suggest that there is no evidence that the model is inconsistent with the data; in other words, the model cannot be rejected. Consequently, when comparing two models, the model that cannot be rejected is preferable to the model that should be rejected given the data.

Another metric for comparing the fit of different models is the Bayesian information (BIC) criterion. The general

Table 6 Mean, SD, and intercorrelations among the variables in Experiment 2 (note that this table reports composite measures of Working Memory and Inhibition)

\begin{tabular}{lccllllll}
\hline & Mean & SD & 1 & 2 & 3 & 5 & 6 \\
\hline 1. Induction Time 1 & 0.63 & 0.21 & 1 & & & \\
2. Induction Time 2 & 0.76 & 0.22 & $0.416^{* *}$ & 1 & & & \\
3. Semantic Space Time 1 & 1.40 & 1.88 & $0.461^{* *}$ & 0.262 & 1 & & \\
4. Semantic Space Time 2 & 2.37 & 2.12 & 0.244 & $0.368^{*}$ & 0.249 & 1 & & \\
5. Working Memory & $\mathrm{z}=0$ & 0.79 & $0.359^{*}$ & $0.625^{* *}$ & $0.365^{*}$ & $0.419^{* *}$ & 1 \\
6. Inhibition & $\mathrm{z}=0$ & 0.82 & 0.053 & $0.325^{*}$ & 0.047 & 0.130 & 0.170 & 1 \\
7. Full-scale IQ & 108.1 & 14.72 & $0.455^{* *}$ & $0.434^{* *}$ & $0.513^{* *}$ & $0.419^{* *}$ & $0.593^{* *}$ & 0.104 \\
\hline
\end{tabular}

*Correlation is significant at the 0.05 level (2-tailed)

**Correlation is significant at the 0.01 level (2-tailed) 


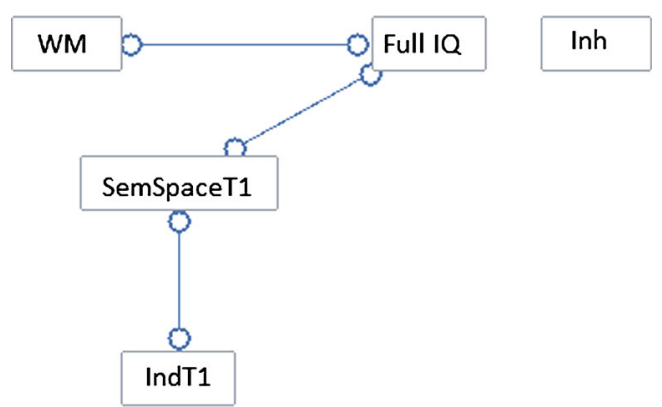

Fig. 5 Set of relations at Time 1 automatically discovered by the FCI search algorithm implemented in TETRAD IV software. WM, composite measure of Working Memory; Full-IQ, Full-scale IQ; Inh, composite measure of Inhibition; SemSpaceT1, difference scores in the Semantic Space task at Time 1; IndT1, Induction scores at Time 1

guidelines for using the BIC criterion are as follows: 1) select the model with the smallest BIC value; 2) BIC differences $<2$ suggest no appreciable differences between the models, whereas BIC differences $>10$ suggest large differences between the models under consideration (Raftery, 1996).

Models 1-3 presented in Fig. 6 are similar in that they are consistent with the data structure discovered by the FCI algorithm and assume a causal link from working memory to fullscale IQ. These models are different in the directionality of the

\section{Model 1}

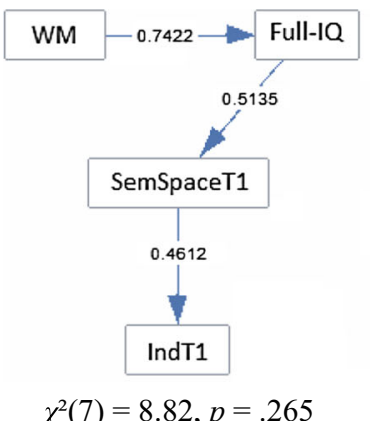

BIC Score $=-17.33$

Model 3

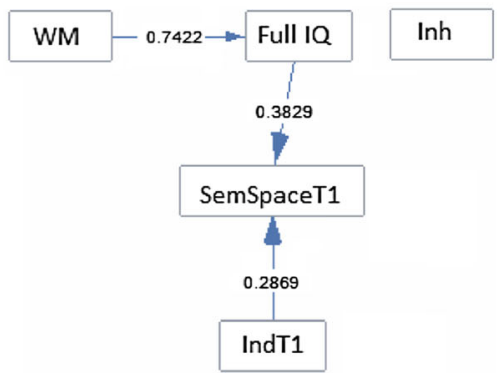

$$
\chi^{2}(7)=14.83, p=.04
$$

BIC Score $=-11.33$

Fig. 6 Graphical representation and statistical summary of a set of models evaluated for Time 1 data. WM, composite measure of Working Memory; Full-IQ, Full-scale IQ; Inh, composite measure of Inhibition; rest of the arrows. Model 1 posits that Full-scale IQ contributes to the Semantic Space scores at Time 1, and these scores in turn affect Induction scores at Time 1 (i.e., IQ $\rightarrow$ Semantic Space $\rightarrow$ Induction). Model 2 reverses the direction of the link between Semantic Space and Full-scale IQ (i.e., Semantic Space $\rightarrow$ IQ) and Model 3 reverses the link between Semantic Space and Induction (i.e., Induction $\rightarrow$ Semantic Space). Finally, Fig. 6 presents Model 4, which is not consistent with the structure automatically discovered by the FCI algorithm but is possible given the correlation matrix in Table 6 (unlike Models 1-3, which are consistent both with the automatically discovered structure and with the correlation matrix). Specifically, Model 4 posits no mediated effects and instead has direct causal links to Induction at Time 1 from all of the variables that were significantly correlated with induction performance (Table 6).

Looking at Fig. 6, Models 2-4 are significantly different from the data, all $\chi^{2} \mathrm{~s}>14.34$ (all $p<0.05$ ) and therefore should be rejected. In contrast, there is no significant difference between the data and Model 1, $\chi^{2}(7)=8.82, p=0.265$. Furthermore, Model 1 has the lowest BIC value (-17.33), which is substantially smaller than the BIC values for Models 2-4 (-11.79, -11.33 , and 9.05 respectively). These results point to the conclusion that only Semantic Space had a direct

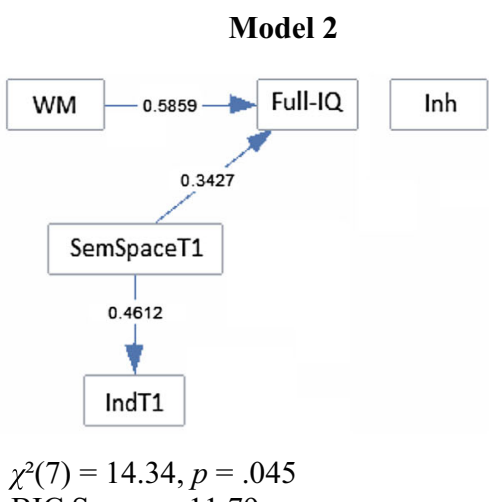

BIC Score $=-11.79$

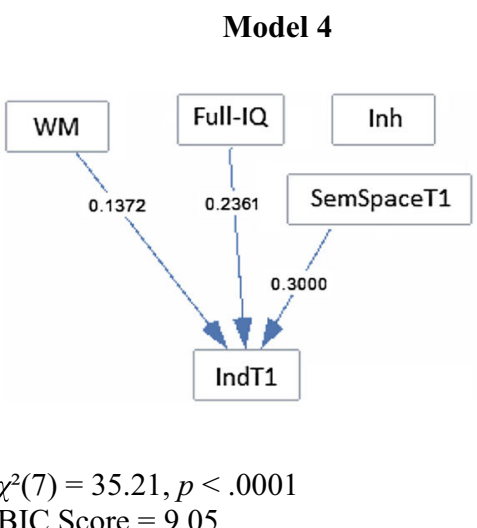

SemSpaceT1, difference scores in the Semantic Space task at Time 1; IndT1, Induction scores at Time 1 
influence on children's Induction scores at Time 1, the influence of Full-scale IQ on Induction was mediated by the Semantic Space scores, and the influence of working memory was mediated by Full-scale IQ through Semantic Space.

Examining the standardized coefficients in Model 1, we can see that if Semantic Space Difference scores are increased by one standard deviation, Induction scores would be expected to increase by 0.46 standard deviations. If Full-scale IQ scores are increased by one standard deviation, Induction scores would be expected to increase by 0.24 standard deviations (because the effect of IQ on Induction is indirect, we need to multiply the standardized coefficients corresponding to the effect of IQ on Semantic Space and effect of Semantic Space On Induction). Finally, if Working Memory is increased by one standard deviation, induction scores would be expected to increase by 0.17 standard deviations (again because the effect of Working Memory on induction is indirect, we need to multiply the three standardized coefficients under consideration).

\section{Identifying possible causal relations at time 2}

Similar to Time 1, we performed automatic search for relations among the variables using the FCI search algorithm using the following variables: Induction scores at Time 1 and Time 2, Full-scale IQ, Semantic Space Differences scores at Time 1 and Time 2, composite Inhibition scores, and composite Working Memory scores. The set of relations discovered by the FCI search algorithm for Time 2 data is shown in Fig. 7. FCI algorithm discovered that children's Induction scores at Time 2 were nonindependent from the Induction scores at Time 1, Working Memory, and Inhibition. A bidirectional arrow between Induction at Time 1 and Time 2 indicates that there is a common unrecorded cause of these variables. Finally, the FCI algorithm discovered that Semantic Space scores at Time 1 influenced Induction at Time $1,{ }^{4}$ and Working Memory and Inhibition influence Induction at Time 2.

Next, we evaluated one parameterized model consistent with the automatically discovered structure (Model 5 in Fig. 8) and one model inconsistent with the discovered structure but consistent with the correlation matrix in Table 6 (Model 6 in Fig. 8). For the latter model, we postulated direct unmediated causal links between Induction scores at Time 2 and all of the variable that were positively correlated with these scores. Model 6 was significantly different from the data, $\chi^{2}(16)=60.36, p<0.0001$, and therefore should be rejected. In contrast, there is no significant difference between the data and Model 5, $\chi^{2}(15)=20.99, p=0.14$. Furthermore,

\footnotetext{
${ }^{4}$ This may seem surprising given that the algorithm was not able to establish the directionality of the relationship between Semantic Space at Time 1 and Induction at Time 1; however, it needs to be noted that addition of more variables (as was the case for Time 2 analyses) aids the search algorithm in discovering the underlying causal structure by adding constraints (for details see Spirtes et al., 2000).
}

SemSpaceT2

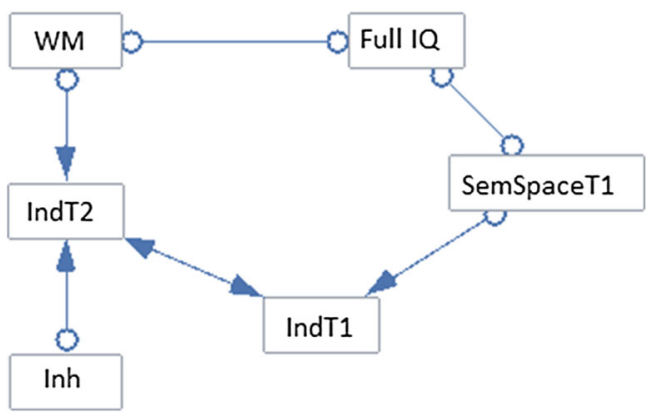

Fig. 7 Set of relations at Time 2 automatically discovered by the FCI search algorithm implemented in TETRAD IV software. WM, composite measure of Working Memory; Full-IQ, Full-scale IQ; Inh, composite measure of Inhibition; SemSpaceT1, difference scores in the Semantic Space task at Time 1; IndT1, Induction scores at Time 1

Model 5 also has a considerably lower BIC score (BIC $=$ -35.07) than Model $6(\mathrm{BIC}=0.56)$.

The above analysis suggests that, unlike at Time 1, children's performance in the Induction task at Time 2 was directly influenced by Working Memory and Inhibition. Examining the standardized coefficients for Model 5, we can see that if Working Memory increased by one standard deviation, we would expect Induction scores at Time 2 to increase by 0.634 standard deviations if we account only for the direct path from Working Memory to Induction (i.e., hold everything else constant) and by 0.672 standard deviations if we account for the total effect of Working Memory on Induction (i.e., the sum of direct and indirect effects). At the same time, if Inhibition increased by one standard deviation, we would expect Induction at Time 2 to increase by 0.277 standard deviations. Additionally, Induction at Time 2 also was influenced by children's performance on this task at Time 1; therefore, Full-scale IQ and Semantic Space Difference scores at Time 1 contributed to children's induction performance at Time 2, with their contribution mediated by children's Induction performance at Time 1 .

\section{General discussion}

The two studies reported here generated a number of novel findings that support the PaRS account of inductive generalization in children. Experiment 1 documented the lure distance effect: preschoolers were more likely to choose a category match item on the induction task in the Distant lure condition than in the Close lure condition. In other words, children's tendency to make category-consistent generalizations was affected not only by the semantic similarity of the targets and category match items but also by the semantic similarity of the targets and lures. 


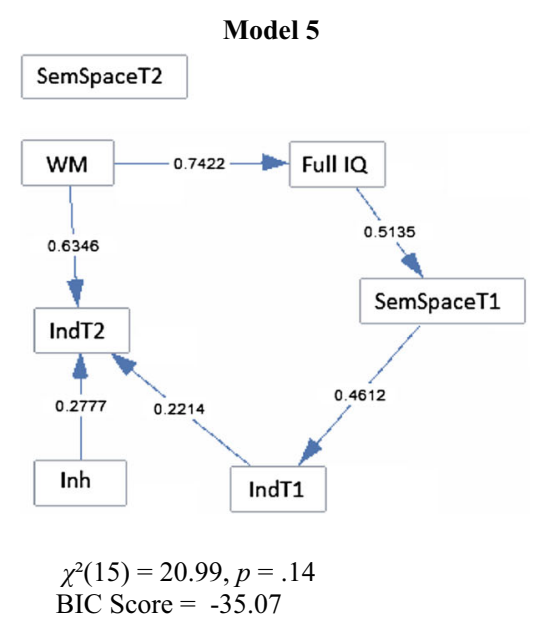

Fig. 8 Graphical representation and statistical summary of a set of models evaluated for Time 2 data. WM, composite measure of Working Memory; Full-IQ, Full-scale IQ; Inh, composite measure of Inhibition;

Experiment 2 is the first longitudinal investigation of the development of children's induction with familiar categories, and it is the first study to explore the role of individual differences in general intelligence, working memory, inhibitory control, and semantic organization in the development of inductive generalization. As such, Experiment 2 reported a number of novel findings pertaining to the development of induction in preschool-age children. In line with previous findings, we initially observed high variability in the individual patterns of responses in 4-year-old children on the induction task: less than a quarter of the sample could be characterized as categoryconsistent responders, with the majority of children being mixed responders. After a 5-month delay, approximately two thirds of children in the sample were category-consistent responders, whereas one third of the children had not yet attained this level of performance. Note that this increase cannot be attributed to children's increased familiarity with the task: the induction task was administered twice within each time point, and we observed no gain in performance from the first to the second administration of the task (i.e., from Time 1a to Time 1b).

At the beginning of the school year, semantic differentiation accounted for most of the variability in children's induction scores: Children who showed moderate semantic differentiation of familiar categories at Time 1 were more likely to make category-consistent inferences with familiar categories than children whose scores on the Semantic Space task suggested low or no differentiation. In other words, when prompted to put animals of similar kind close together, children who placed the alligator closer to the crocodile than to the fish and grasshopper were more likely to make category-based inferences with familiar categories than children who placed the alligator as close to the crocodile as to the fish and grasshopper. Furthermore, among children who were not yet categoryconsistent responders at the beginning of the school year, only

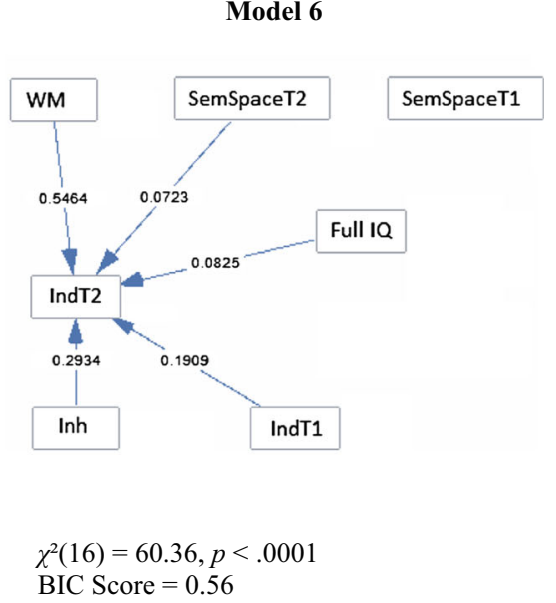

SemSpaceT1, difference scores in the Semantic Space task at Time 1; IndT1, Induction scores at Time 1

those children who showed improved semantic differentiation by the end of the school year also showed increased categoryconsistent responding on the induction task.

Results of the Structural Equation Modeling (SEM) suggested that at Time 1 there was a sizable $(\beta=0.461)$ direct (unmediated) contribution of semantic differentiation to children's performance in the induction task. Additionally, the analyses suggested that individual differences in general intelligence and working memory also contributed to induction performance at Time 1; however, these influences were indirect: the influence of general intelligence on induction was mediated through semantic differentiation and the influence of working memory was mediated through general intelligence.

A moderate degree of semantic differentiation may be necessary but not sufficient to make category-consistent inferences on the induction task used in this study. Specifically, we hypothesized that two additional processes may play a role in children's performance on the induction task: working memory (needed for active maintenance of task-relevant representations) and inhibitory control (needed to select the appropriate representation among several competing activated representations). Analyses of Time 2 data provided strong evidence that working memory contributed to children's induction performance and moderate evidence for the role of inhibitory control. Specifically, individual differences in working memory accounted for most of the variance in induction scores at Time 2. Furthermore, SEM analysis suggested that in the beginning of the school year (when most children in the sample exhibited low semantic differentiation), working memory made no direct contribution to children's induction performance. In contrast, at the end of the school year (when most children exhibited moderate semantic differentiation), there was a sizable $(\beta=0.63)$, direct (unmediated) contribution of working memory to performance in the induction task. This analysis also suggested a direct contribution of inhibitory 
control, although this contribution was considerably weaker $(\beta=0.28)$. In other words, individual differences in working memory and inhibitory control made a direct contribution to children's induction performance only when there was a moderate degree of semantic differentiation.

\section{Theoretical implications}

The reported findings support the predictions of the PaRS account put forth in this paper. Specifically, Experiment 1 provided evidence that semantic differentiation plays an important role in inductive generalization with familiar categories in 4-yearold children; Experiment 2 provided evidence that increased semantic differentiation is related to the emergence of categoryconsistent inferences in young children. At the same time, the reported findings are not fully consistent with the extant theories of inductive generalization early in development.

Specifically, SINC can account for children's performance in the Close Lure condition of Experiment 1 and Induction scores at Time 1 in Experiment 2. In both cases, the majority of 4-year-old children did not provide category-consistent responses. This pattern of results is expected if only perceptual similarity influences induction and no useful perceptual information is provided (as was the case in the present studies). However, SINC cannot account for the emergence of category-consistent responses at Time 2 in Experiment 2 or category-consistent responses in the Distant Lure condition in Experiment 1 . In contrast, the naïve theory approach can explain children's successful induction performance in the Distant Lure condition of Experiment 1 and at Time 2 in Experiment 2. However, the naïve theory approach is inconsistent with the majority of children being mixed responders in the Close Lure condition of Experiment 1 and at Time 1 in Experiment 2. Finally, neither of the extant accounts makes predictions relating an increase in semantic differentiation to emergence of category-consistent inferences.

Whereas the reported findings are particularly problematic for SINC, it could be argued that these findings do not rule out the possibility that children's inferences were not only category-consistent in their outcome - in the Far Lure condition in Experiment 1 and at Time 2 in Experiment 2-but also were category-based in the mechanism by which they were generated. However, unlike PaRS, the category-based approach does not provide an account of developmental change in inductive generalization reported here and in prior studies. Nevertheless, future research is necessary to distinguish between these possibilities.

\section{Limitations and outstanding questions}

One limitation of the reported findings is a relatively small sample size in Experiment 2. However, the reported results provide the first clear evidence that (1) there are stable individual differences in children's inductive generalization with familiar categories and that (2) individual differences in inductive generalization are related to individual differences in general cognitive functioning as well as semantic development. These results mandate further study of the relationship between inductive generalization and individual differences in a number of cognitive processes. The present results also strongly suggest that individual difference factors not examined in the present study likely contribute to the development of inductive generalization. Therefore, it is important to identify and evaluate additional individual difference factors in future research.

Recall that the PaRS account draws a distinction between two sources of similarity information: featural overlap in perceptual input (i.e., perceptual similarity) and featural overlap in semantic memory (i.e., representational similarity). When one makes an inference about a member of a novel category, perceptual similarity is the only source of information that can contribute to the inference. However, when one makes an inference about a member of a familiar category, the inference is influenced both by the overlap in observable features and features stored in memory. Semantic development undoubtedly includes an increase in the number of features of familiar items that are stored in memory; this increase is widely hypothesized to drive developmental changes in representational similarity (Hills et al., 2009; Kemp \& Tenenbaum 2008; Rogers \& McClelland, 2004). The present findings support the PaRS framework by providing evidence that developmental changes in representational similarity can help explain developmental changes in inductive generalization with familiar categories. At present, the account does not specify the relative contribution of representational and perceptual similarity to induction. One possibility is that developmental changes in induction stem not only from changes in representational similarity, but also from changes in the contribution that perceptual and representational similarity make to induction. For example, it is possible that in the course of development the contribution of perceptual similarity decreases, whereas the contribution of representational similarity increases. This possibility remains to be tested in future research.

Finally, the present studies suggest that developmental changes in representational similarity contribute to the development of category-consistent inferences with familiar categories; however, these studies do not elucidate the origins of developmental change in representational similarity. As stated above, one widely hypothesized source of change in representational similarity is an increase in the number of known features of familiar categories. Therefore, exposure to different sources of information (e.g., going to the zoo or a museum, traveling, reading or being read to, etc.) is one likely source of developmental change in representational similarity. However, exposing different children to the same information is unlikely to result in equivalent learning and equivalent changes 
to semantic structure. Our current hypothesis is that individual differences in short-term memory span and long-term storage can help to explain individual differences in semantic structure when exposure to new content is held constant. Further research is needed to evaluate this hypothesis and explain developmental changes in representational similarity.

\section{Conclusions}

In this paper, we put forth the PaRS account of the development of inductive generalization with familiar categories and provided evidence from two experiments in support of this new account. In contrast to the existing theories, PaRS is the first truly developmental account of induction, because it explains developmental changes in children's inductive generalization with familiar categories.

Acknowledgments The authors thank the children, parents, teachers, and administrators of the Children's School at Carnegie Mellon University for making this work possible, and also thank Malika Sinha, Laura Pacilio, Alyssa Montanaro, Anna Loiterstein, Like Li, Kayoung Joung, Jennifer Shin, and Rachel Walsh for their help collecting data. Thank you to Vladimir Sloutsky, Erik Thiessen, and David Rakison for providing comments on an earlier version of this manuscript. This work was supported in part by a Graduate Training Grant awarded to Carnegie Mellon University by the Department of Education (R305B090023 and R305B040063). The opinions expressed are those of the authors and do not represent views of the Department of Education.

\section{Appendix}

To reduce the number of observed variables ostensibly probing the same latent variables, we performed a Principal Axis Factor (PAF) analysis with a Varimax (orthogonal) rotation of the following six individual difference measures: FWS, BWS, memory accuracy on the Track-It task, Flanker task Accuracy Difference scores, and accuracy scores on the Day/Night task. Kaiser-Meyer Olkin (KMO) test of sampling adequacy suggested that the sample size was acceptable to perform factor analysis, $\mathrm{KMO}=0.665$ (values $>0.50$ are considered acceptable). Bartlett's test of sphericity indicated that non-zero correlations in the sample matrix were unlikely to stem from sampling error and therefore, the matrix was factorable, Bartlett's $\chi^{2}(10)=46.463, p<0.0001$.

The results of an orthogonal rotation of the solution are shown in the table below. The final solution extracted two factors, which cumulatively accounted for $72.66 \%$ of the observed variance. As shown in the table, scores on the FWS and Track-It tasks correlated highly with Factor 1 (factor loadings of 0.814 and 0.854 , respectively) and negligibly with Factor 2 (factor loadings of -0.126 and 0.056 , respectively). The BWS measure had nontrivial correlations with both factors but higher factor loading on Factor 1 than on Factor 2 (0.693 and 0.493, respectively). In contrast, the Day/Night measure and Flanker Difference scores were strongly correlated with Factor 2 (factor loadings of 0.767 and 0.790 , respectively) and less so with Factor 1 (factor loadings of 0.391 and -0.364 , respectively). These results suggest that Factor 1 can be interpreted as Working Memory and Factor 2 can be interpreted as Inhibition, a solution that is consistent with studies of the latent structure of executive functions in adults (Miyake et al., 2000) and in children (Lehto, Juujärvi, Kooistra, \& Pulkkinen, 2003).

Based on the outcome of the exploratory factor analysis, we calculated a composite measure of Working Memory and Inhibition by first transforming the raw scores into z-scores and then averaging the z-scores across the FWS, BWS, and Track-It tasks to obtain a composite measure of Working Memory, and across the Day/Night task and the Flanker task to obtain a composite measure of Inhibition. These composite scores were used in the analyses reported in Part III of the Analysis of Experiment 2.

Obliquely rotated component loadings for the six EF measures in Experiment 2

\begin{tabular}{lcc}
\hline & Component & \\
\hline & 1 & 2 \\
FWM & 0.814 & -0.126 \\
BWM & 0.693 & 0.493 \\
Track-It task & 0.854 & 0.056 \\
Day/Night task & 0.391 & 0.767 \\
Flanker Difference scores & -0.364 & 0.790 \\
\hline
\end{tabular}

\section{References}

Akshoomoff, N. A. (2002). Selective attention and active engagement in young children. Developmental Neuropsychology, 22, 625-642.

Badger, J. R., \& Shapiro, L. R. (2012). Evidence of a transition from perceptual to category induction in 3- to 9-year-old children. Journal of Experimental Child Psychology, 113, 131-146.

Badre, D., \& Wagner, A. D. (2002). Semantic retrieval, mnemonic control, and prefrontal cortex. Behavioral and Cognitive Neuroscience Reviews, 1(3), 206-218.

Baldwin, D. A., Markman, E. M., \& Melartin, R. L. (1993). Infants' ability to draw inferences about nonobvious object properties: Evidence from exploratory play. Child Development, 64, 711-728.

Best, J. R., \& Miller, P. H. (2010). A developmental perspective on Executive Function. Child Development, 81, 1641-1660. doi:10. 1111/j.1467-8624.2010.01499.x

Bomba, P. C., \& Siqueland, E. R. (1983). The nature and structure of infant form categories. Journal of Experimental Child Psychology, 35, 294-328.

Booth, A. E., \& Waxman, S. R. (2002). Word learning is 'smart': evidence that conceptual information affects preschoolers' extension of novel words. Cognition, 84, 11-22. 
Carlson, S. M. (2005). Developmentally sensitive measures of executive function in preschool children. Developmental Neuropsychology, 28, 595-616.

Chi, M. T. H., Hutchinson, J., \& Robin, A. F. (1989). How inferences about novel domain-related concepts can be constrained by structured knowledge. Merrill-Palmer Quarterly, 35, 27-62.

Chen, C., \& Stevenson, H. W. (1988). Cross-linguistic differences in digit span of preschool children. Journal of Experimental Child Psychology, 46, 150-158.

Clark, E. V. (1973). What's in a word? On the child's acquisition of semantics in his first language. In T. E. Moore (Ed.), Cognitive Development and the Acquisition of Language (pp. 65-110). New York: Academic Press.

Dale, P. S., \& Fenson, L. (1996). Lexical development norms for young children. Behavior Research Methods, Instruments \& Computers, 28, 125-127.

Diamond, A. (2002). Normal development of prefrontal cortex from birth to young adulthood: Cognitive functions, anatomy, and biochemistry. In D. T. Stuss \& R. T. Knight (Eds.), Principles of frontal lobe function (pp. 466-503). London, UK: Oxford University Press.

Dunn, L. M., \& Dunn, L. M. (1997). Peabody Picture Vocabulary Test (3rd ed.). Circle Pines, MN: AGS Publishing.

Fisher, A. V. (2007). Are developmental theories of learning paying attention to attention? Cognition, Brain, and Behavior, 11, 635-646.

Fisher, A. V. (2011). Processing of perceptual information is more robust than processing of conceptual information in preschool-age children: Evidence from costs of switching. Cognition, 119, 253-264.

Fisher, A. V., \& Sloutsky, V. M. (2005). When induction meets memory: Evidence for gradual transition from similarity-based to categorybased induction. Child Development, 76, 583-597.

Fisher, A. V., Matlen, B. J., \& Godwin, K. E. (2011). Semantic similarity of labels and inductive generalization: Taking a second look. Cognition, 118, 432-438.

Fisher, A. V., Thiessen, E. D., Godwin, K., Kloos, H., \& Dickerson, J. P. (2013). Assessing selective sustained attention in 3- to 5-year-old children: Evidence from a new paradigm. Journal of Experimental Child Psychology, 114, 275-294.

Fisher, A. V., Godwin, K. E., Matlen, B. J., \& Unger, L. (2014). Development of category-based induction and semantic knowledge. Child Development. doi:10.1111/cdev.12277

French, R. M., Mareschal, D., Mermillod, M., \& Quinn, P. C. (2004). The role of bottom-up processing in perceptual categorization by 3 - to $4-$ month-old infants: Simulations and data. Journal of Experimental Psychology: General, 133, 382-397.

Gelman, S. A. (2003). The essential child: Origins of essentialism in everyday thought. New York: Oxford University Press.

Gelman, S. A., \& Davidson, N. S. (2013). Conceptual influences on category-based induction. Cognitive Psychology, 66, 327-353.

Gelman, S. A., \& Coley, J. D. (1990). The importance of knowing a Dodo is a bird: Categories and inferences in 2-year-old children. Developmental Psychology, 26, 796-804.

Gelman, S. A., \& Markman, E. (1986). Categories and induction in young children. Cognition, 23, 183-209.

Gelman, S. A., \& Wellman, H. M. (1991). Insides and essences: Early understandings of the non-obvious. Cognition, 38, 213-244.

Gentner, D. (1978). A study of early word meaning using artificial objects: What looks like a jiggy but acts like a zimbo? Papers and Reports on Child Language Development, 15, 1-6, Stanford University.

Gerstadt, C. L., Hong, Y. J., \& Diamond, A. (1994). The relationship between cognition and action: performance of children $31 / 2-7$ years old on a Stroop-like day-night test. Cognition, 53, 129-153.

Godwin, K. E., Matlen, B., \& Fisher, A. V. (2013). Development of category-based reasoning in 4- to 7-year-old children: The influence of label co-occurrence and kinship knowledge. Journal of Experimental Child Psychology, 115, 74-90.
Goldstone, R. (1994). An efficient method for obtaining similarity data. Behavior Research Methods, Instruments, \& Computers, 26, 381386.

Graham, S. A., Booth, A., \& Waxman, S. R. (2012). Words are not merely features: Only consistently applied nouns guide 4-year-olds' inferences about object categories. Language Learning and Development, 8, 1-11.

Graham, S. A., Kilbreath, C. S., \& Welder, A. N. (2004). Thirteen-montholds rely on shared labels and shape similarity for inductive inferences. Child Development, 75, 409-427.

Hills, T. T., Maouene, M., Maouene, J., Sheya, A., \& Smith, L. B. (2009). Categorical structure among shared features in networks of earlylearned nouns. Cognition, 112, 381-396. doi:10.1016/j.cognition. 2009.06.002

Howard, D. V., \& Howard, J. H., Jr. (1977). A multidimensional scaling analysis of the development of animal names. Developmental Psychology, 13, 108-113.

Inagaki, K. (1990). The effects of raising animals on children's biological knowledge. British Journal of Developmental Psychology, 8(2), $119-129$.

Jaswal, V. K., \& Markman, E. M. (2007). Looks aren't everything: 24month-olds' willingness to accept unexpected labels. Journal of Cognition and Development, 8, 93-111.

Jones, S. S., \& Smith, L. B. (1998). How children name objects with shoes. Cognitive Development, 13, 323-334.

Kan, I. P., \& Thompson-Schill, S. L. (2004). Selection from perceptual and conceptual representations. Cognitive, Affective, \& Behavioral Neuroscience, 4, 466-482.

Kemp, C., \& Tenenbaum, J. B. (2008). The discovery of structural form. Proceedings of the National Academy of Sciences, 105, 1068710692. doi:10.1073/pnas.0802631105

Krawczyk, D. C., Morrison, R. G., Viskontas, I. V., Holyoak, K. J., Chow, T. W., Mendez, M., ... Knowlton, B. J. (2008). Distraction during relational reasoning: The role of prefrontal cortex in interference control. Neuropsychologia, 46, 2020-2032.

Lehto, J. E., Juujärvi, P., Kooistra, L., \& Pulkkinen, L. (2003). Dimensions of executive functioning: Evidence from children. British Journal of Developmental Psychology, 21, 59-80.

Long, C., Lu, X., Zhang, L., Li, H., \& Deák, G. O. (2011). Category label effects on Chinese children's inductive inferences: Modulation by perceptual detail and category specificity. Journal of Experimental Child Psychology, 111, 230-245. doi:10.1016/j.jecp.2011.08.010

Lowe, W. (1997). Meaning and the mental lexicon. Proceedings of the 15th International Joint Conference on Artificial Intelligence, pp. 1092-1097. San Francisco: Morgan Kaufmann.

Markman, E. M. (1989). Categorization and naming in children: Problems of induction. Cambridge, MA: MIT Press, Bradford Books.

Matlen. B. J., Fisher, A. V., \& Godwin, K. E. (under review). The influence of label co-occurrence on inductive generalization in preschool-age children.

Miller, E. K., \& Cohen, J. D. (2001). An integrative theory of prefrontal cortex function. Annual Review of Neuroscience, 24, 167-202.

Miyake, A., Freidman, N. P., Emerson, M. J., Witzki, A. H., Howerter, A., \& Wager, T. D. (2000). The unity and diversity of executive functions and their contributions to complex 'frontal lobe' tasks: a latent variable analysis. Cognitive Psychology, 41, 49-100.

Morrison, R. G., Doumas, L. A. A., \& Richland, L. E. (2011). A computational account of children's analogical reasoning: Balancing inhibitory control in working memory and relational representation. Developmental Science, 14(3), 516-529.

Noles, N. S., \& Gelman, S. A. (2012). Effects of categorical labels on similarity judgments: A critical analysis of similarity-based approaches. Developmental Psychology, 48, 890-896. doi:10.1037/ a0026075 
Opfer, J. E., \& Bulloch, M. J. (2007). Causal relations drive young children's induction, naming, and categorization. Cognition, 105, $207-$ 217

Osherson, D., Smith, E., Wilkie, O., Lopez, A., \& Shafir, A. (1990). Category-based induction. Psychological Review, 97, 185-200.

Raftery, A. E. (1996). Bayesian model selection in social research. In P. V. Marsden (Ed.), Sociological Methodology (Vol. 25, pp. 111-163). Oxford: Basil Blackwell.

Rakison, D. H., \& Poulin-Dubois, D. (2002). You go this way and I'll go that way: Developmental changes in infants' attention to correlations among dynamic features in motion events. Child Development, 73, 682-699.

Richland, L. E., Morrison, R. G., \& Holyoak, K. J. (2006). Children's development of analogical reasoning: Insights from scene analogy problems. Journal of Experimental Child Psychology, 94, 249-273.

Rogers, T. T., \& McClelland, J. L. (2004). Semantic Cognition: A Parallel Distributed Processing Approach. Cambridge, MA: MIT Press.

Rueda, M. R., Fan, J., McCandliss, B. D., Halparin, J. D., Gruber, D. B., Lercari, L. P., \& Posner, M. (2004). Development of attentional networks in childhood. Neuropsychologia, 42(8), 1029-1040.

Scheines, R., Spirtes, P., Glymour, C., Meek, C., \& Richardson, T. (1998). The TETRAD Project: Constraint Based Aids to Causal Model Specification. Multivariate Behavioral Research, 33, 65-117.

Simpson, A., \& Riggs, K. J. (2005). Inhibitory and working memory demands of the day-night task in children. British Journal of Developmental Psychology, 23, 471-486.

Sloman, S. A. (1993). Feature-based induction. Cognitive Psychology, $25,231-280$.

Sloutsky, V. M. (2010). From perceptual categories to concepts: What develops? Cognitive Science, 34, 1244-1286.

Sloutsky, V. M., \& Fisher, A. V. (2004). Induction and categorization in young children: A similarity-based model. Journal of Experimental Psychology: General, 133, 166-188. doi:10.1037/0096-3445.133.2. 166

Sloutsky, V. M., \& Fisher, A. V. (2012a). Linguistic labels: Conceptual markers or object features? Journal of Experimental Psychology, 111, 65-86. doi:10.1016/j.jecp.2011.07.007
Sloutsky, V. M., \& Fisher, A. V. (2012b). Effects of categorical labels on similarity judgments: A critical evaluation of a critical analysis. Comment on Noles and Gelman. Developmental Psychology, 48, 987-900.

Sloutsky, V. M., Lo, Y.-F., \& Fisher, A. (2001). How much does a shared name make things similar? Part 2: Linguistic labels and the development of inductive inference. Child Development, 72, 1695-1709.

Sloutsky, V. M., Kloos, H., \& Fisher, A. V. (2007). When looks are everything: Appearance similarity versus kind information in early induction. Psychological Science, 179-185. doi: 10.1111/j.14679280.2007.01869

Smith, L. B., Jones, S. S., \& Landau, B. (1996). Naming in young children: a dumb attentional mechanism? Cognition, 60, 143-171.

Smith, L. B., Jones, S. S., Yoshida, H., \& Colunga, E. (2003). Whose DAM account? Attentional learning explains. Cognition, 87, 209 213.

Spirtes, P. (2001). An anytime algorithm for causal inference. In T. Jaakkola \& T. Richardson (Eds.), Proceedings of International Workshop on Artificial Intelligence and Statistics, pp. 213-231.

Spirtes, P., Glymour, C., \& Scheines, R. (2000). Causation, Prediction, and Search. New York, N.Y.: MIT Press.

Tulsky, D. S., Carlozzi, N. E., Chevalier, N., Espy, K. A., Beaumont, J. L., \& Mungas, D. (2013). NIH toolbox cognition battery (CB): Measure of working memory. In P. D. Zelazo and P. Bauer (Eds.), National Institutes of Health Toolbox Cognition Battery (NIH Toolbox CB): Validation for Children Between 3 and 15 Years. Monographs of the Society for Research in Child Development, 78, 70-87.

Quinn, P. C., Eimas, P. D., \& Rosenkratz, S. L. (1993). Evidence for representations of perceptually similar natural categories by 3 month-old and 4-month-old infants. Perception, 22, 463-475.

Unger, L., Fisher, A. V., \& MacLellan, C. J. (2014). Developmental changes in semantic organization of living kinds. In P. Bello, M. Guarini, M. McShane, \& B. Scassellati (Eds.), Proceedings of the 36th Annual Meeting of the Cognitive Science Society, 1646-1651.

Wechsler, D. (1989). Wechsler Preschool and Primary Scale of Intelligence - Revised. San Antonio, TX: The Psychological Corporation. 